\title{
Improved accuracy and precision of bioprinting through progressive cavity pump-controlled extrusion
}

\section{Journal Article}

\section{Author(s):}

Fisch, Philipp; Holub, Martin; Zenobi-Wong, Marcy (D)

Publication date:

2021-01

Permanent link:

https://doi.org/10.3929/ethz-b-000458795

Rights / license:

Creative Commons Attribution 4.0 International

Originally published in:

Biofabrication 13(1), https://doi.org/10.1088/1758-5090/abc39b

Funding acknowledgement:

173868 - Sinergia Project: A Tissue, Cell and Molecular Approach to Understanding and Treating Microtia (SNF) 
1 Improved accuracy and precision of bioprinting through progressive cavity pump-controlled extrusion

\author{
Philipp Fisch ${ }^{1}$, Martin Holub ${ }^{1,2}$ and Marcy Zenobi-Wong ${ }^{1}$ \\ ${ }^{1}$ Department of Health Science and Technology, ETH Zurich, Zurich, Switzerland \\ ${ }^{2}$ Present address: Department of Bionanoscience, TU Delft, Delft, Netherlands \\ E-mail: marcy.zenobi@hest.ethz.ch
}

Received xxxxxx

Accepted for publication $\mathrm{xxxxxx}$

Published xxxxxxAbstract

3D bioprinting has seen a tremendous growth in recent years in a variety of fields such as tissue engineering, drug testing and regenerative medicine, which has led researchers and manufacturers to continuously advance and develop novel bioprinting techniques and materials. Although new bioprinting methods are emerging (e.g. contactless and volumetric bioprinting), micro-extrusion bioprinting remains the most widely used method. Micro-extrusion bioprinting, however, is still largely dependent on the conventional pneumatic extrusion process, which relies heavily on homogenous biomaterial inks and bioinks to maintain a constant material flow rate. Augmenting the functionality of the bioink with the addition of nanoparticles, cells or biopolymers can induce inhomogeneities resulting in uneven material flow during printing and/or clogging of the nozzle, leading to defects in the printed construct. In this work, we evaluated a novel extrusion technique based on a miniaturized progressive cavity pump which allows precise control over the volumetric flow rate by positive displacement. We compared the accuracy and precision of this system to the pneumatic extrusion system and tested both systems for their effect on cell viability after extrusion. The progressive cavity pump achieved a significantly higher accuracy and precision compared to the pneumatic system, while maintaining good viability. These improvements were independent of the bioink composition, printing speed or nozzle size. This study demonstrates the merit of precise extrusion-process control in bioprinting by progressive cavity pumps and investigates their influence on process-induced cell damage. Progressive cavity pumps are a promising tool for bioprinting and could help provide standardized and validated bioprinted constructs while leaving the researcher more freedom in the design of the bioinks.

Keywords: bioprinting, progressive cavity pump

\section{Introduction}

3D bioprinting with its potential to position materials and cells in a precise 3-dimensional arrangement has gained growing interest for use in tissue and organ models, drug testing and regenerative medicine, leading to a tremendous growth in the bioprinting industry. (1) Starting from the first inkjet bioprinter, a modified HP 660C printer, the technology has evolved continuously and users can nowadays choose from a variety of commercially available bioprinting technologies. (2-4)
These technologies can be categorized based on 4 main
strategies: (i) laser induced forward transfer (ii) dropletbased bioprinting, (iii) extrusion-based bioprinting and (iv) stereolithography-based bioprinting, each of which can be further sub-categorized based on the exact mechanisms used to position materials and cells. (1) Among these, extrusion-based bioprinting is the most commonly used process to fabricate tissues and organs, mainly because of its ease of use, scalability and wide availability of printable materials. (5) Extrusion printing functions by positive displacement of material using a 
plunger which is either driven by pneumatic pressure (pneumatic extrusion) or by a piston which displaces the plunger (piston driven extrusion). Another method frequently referred to in literature is the use of an Auger screw which transports material from a feedstock to the nozzle, but except for a recent publication by Ning et al. (6) has only been described in the context of conventional fused deposition modeling 3D printing (3, 7-9).

Despite the ability of extrusion-based bioprinting to deposit a wide range of hydrogel precursors, great care in the composition and preparation of the printing materials is required to obtain the rheological and biological characteristics needed for excellent printability and biocompatibility. (10) Furthermore, additional factors such as the mixing of the bioink or the removal of air bubbles are critical, as uneven flow and nozzle clogging due to inhomogeneities in the bioink are a commonly observed phenomenon to which pneumatic extrusion systems are particularly susceptible. $(1,11)$ Especially highly viscous biomaterial inks and bioinks containing viscosifiers as well as micro- and nano-composite bioinks are difficult to print. The structural integrity as well as the shape fidelity of the bioprinted construct cannot be guaranteed without online adjustment of the pressure to compensate for variations in the volume flow. This becomes important for clinical applications where regulatory authorities will demand standardization of the printing process. (12)

Progressive cavity pumps (PCPs) offer an alternative to the aforementioned pneumatic extrusion-bioprinting process (Figure $1 \mathrm{~A}$ ). Initially used in heavy industry for purposes such as artificial lifting in the oil industry, mining operations or food processing, their miniaturization opened up applications for adhesives and sealants, medical devices and 3D printing. (13-15) Recently their use in 3D printing of materials incorporating complex compositional and mechanical gradients using a two-component PCP system has been explored. $(16,17)$ The extrusion process of PCPs is governed by a single-helical rotor which rotates eccentrically within a double-helical stator of twice the pitch length (Figure $1 \mathrm{~B}$ ). Between the seal lines (the contact lines of rotor and stator), cavities are created which are constantly moved towards the discharge end of the pump. As one cavity is eliminated, another cavity develops, keeping the cross-sectional area of the cavities constant, leading to a continuous non-pulsating flow. (18) The pushing-and-suction action therefore allows PCPs to exert significantly lower shear rates on the material compared to other pump types, such as membrane pumps or peristaltic pumps. Additionally, by knowing the geometry of the rotor and stator, the flow rate and deposited volume can be extremely accurately controlled by the rotational speed of the rotor, e.g. down to $1 \mu \mathrm{l}$. (19) The successful application of PCPs for 3D printing of biological materials has been shown by printing bacterial spores to create biohybrid films $(20,21)$ and by printing bacteria in precise 3-dimensional shapes for biomedical applications. (22)

This study further extends the application of progressive cavity pumps to the field of bioprinting and demonstrates how precise extrusion-process control can facilitate the reproducible manufacturing of tissue engineered constructs. We compare the most commonly used pneumatic extrusion process to a miniaturized PCP in terms of their accuracy and precision as well as the process-related cell damage (Figure $1 \mathrm{~A}$ ). We utilized an alginate-gellan gum bioink as model bioink $(23,24)$ and further explored the capabilities of PCPs using two previously reported bioinks based on either gelatin and alginate (25) or gelatin methacryloyl (GelMA) and Laponite (LPN) (26). By combining alginate and gellan gum, a bioink with tunable shear thinning and shear recovery behavior can be created based on the formation of double helices by gellan gum upon cooling. $(27,28)$ The bioink furthermore offers a platform for various types of tissue through the addition of different ECM particles, thereby extending its tissue specific bioactivity.(23) Gelatin and GelMA are widely used biomaterials due to their similarity to the extracellular matrix (ECM) providing cell-adhesive and proteolytic peptide motifs and tunable mechanical properties. (29-31) LPN further enhances the GelMA bioink by improving its printability as well as by providing biofunctionality by clay-protein interaction. (26) Nonetheless, as gelatin and GelMA undergo thermal gelation during cooling, these bioinks either require a temperature controlled extrusion process, (32) have to be printed during a specific time window with continuous pressure adjustment (25) or require the use of a piston driven extrusion process (26).

In this study, we tested the sensitivity of the extrusion process to increased polymer content, addition of hydroxyapatite particles, commercial source of alginate based on the alginate-gellan gum bioink (Figure $1 \mathrm{C}$, Bioink 1-4) and using gelatin-based bioinks (Figure $1 \mathrm{D}$, Bioink 5-6). The accuracy and precision of the two methods were determined by comparing the actual weight of the printed constructs compared to the targeted weight, after both printing processes were optimized. To further test the boundaries of the PCP extrusion, we studied if the accuracy and precision could be maintained when increasing nozzle diameter and printing speed (Figure 1 E). Next, we evaluated the performance of the PCP when 
1 printing complex structures with intermittent printing 2 paths such as the ear and nose with the alginate-gellan 3 gum bioink as well as the gelatin and GelMA bioinks 4 (Figure $1 \mathrm{~F}$ ). Lastly, to evaluate the effect on cell viability, constructs were cast and bioprinted with the pneumatic system or the PCP, with and without a printing nozzle, and cell viability analyzed directly, one and 14 days after printing (Figure $1 \mathrm{G}$ ). By providing these novel insights on progressive cavity pumps and relevant information on 0 their extrusion process for bioprinting, this study 11 advances the toolset of extrusion methods needed for the 12 standardization of bioprinted constructs.

\section{Methods}

Bioprinting was performed on a Biofactory bioprinte (regenHU, Switzerland). Printing files were either prepared in slic3r (https://slic3r.org/) and post-processed in a custom written Matlab postprocessor (Matlab 2018a, Mathworks) or prepared in BioCAD (regenHU, Switzerland).

\subsection{Progressive cavity pump (PCP) installation and preparation}

The eco-PEN300 PCP (preeflow by ViscoTec GmbH) was installed in the Biofactory with a custom 3D printed mount (Supplementary Figure 1 D-F) and controlled by the eco-CONTROL EC200-K unit. As start/stop signal, the $24 \mathrm{~V}$ pressure on/off signal of the DD-135N Biofactory printhead was connected to the ecoCONTROL unit.

To prepare the PCP for printing, the bioink cartridge was connected to the PCP by a Luer lock adapter allowing material flow into the cavity ahead of the rotor/stator of the PCP. The cartridge was connected to the pressure valve and regulator of the bioprinter allowing material flow into the PCP. To vent the cavity, the bleed screw of the PCP was opened, pressure applied to the plunger of the bioink cartridge and material allowed to flow out until free of air bubbles. Afterwards, the bleed screw was closed and the PCP started until material started to flow out of the nozzle. Before each test, the PCP was calibrated by extruding a specific amount of material, weighing it, converting the measured weight to volume and comparing the extruded volume to the expected volume.

Proper calibration was ensured by extruding $50 \mathrm{mg}$ at $100 \mu \mathrm{l} / \mathrm{min}, 150 \mu \mathrm{l} / \mathrm{min}$ and $200 \mu \mathrm{l} / \mathrm{min}$ and weighing the extrudate (the minimum volume flow in the technical specifications of the eco-PEN300 PCP is $120 \mu \mathrm{l} / \mathrm{min}$ ). Achievable volume flows of the pneumatic system for comparison are: $76.4 \pm 9.9 \mu \mathrm{l} / \mathrm{min}(20 \mathrm{kPa}), 129.1 \pm 7.5$ $\mu \mathrm{l} / \mathrm{min}(25 \mathrm{kPa})$, and $212.8 \pm 14.9 \mu \mathrm{l} / \mathrm{min}(30 \mathrm{kPa})$. The extrudate of three different batches of bioink was collected and the test repeated three times for each batch.

\subsection{GelMA synthesis}

GelMA was synthesized as reported previously. (26) Briefly, $10 \mathrm{~g}$ of gelatin (type A, porcine, 300 bloom, Sigma) were dissolved in PBS at $50^{\circ} \mathrm{C}$ under magnetic stirring for $1 \mathrm{~h}$. Following, $0.1 \mathrm{ml}$ per g gelatin of methacrylic anhydride was added dropwise under stirring and the reaction allowed to proceed for $1 \mathrm{~h}$. The reaction was stopped by $2.5 \mathrm{x}$ dilution, subsequently centrifuged at $3000 \mathrm{rcf}$ and then dialyzed against ultrapure water at $40^{\circ} \mathrm{C}$ for 5 days. A degree of methacrylation of $30 \%$ was determined by $1 \mathrm{H}$-proton nuclear magnetic resonance spectroscopy (Bruker Advance $400 \mathrm{MHz}$ ) according to (33).
66

\subsection{Bioink preparation}

Gellan gum and alginate bioinks were prepared by dissolving gellan gum (Kelcogel GG-LA [GG], CP Kelco) and alginate (Algin I-1, Kimica $\left[\mathrm{Alg}_{K}\right.$ ] or Pronova

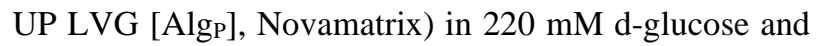
$20 \mathrm{mM}$ HEPES, $\mathrm{pH} 7.4$, at $90^{\circ} \mathrm{C}$ for $1 \mathrm{~h}$ while stirring. Similarly, the gellan gum, alginate and hydroxyapatite particle (HAp, Acros Organics, 1-10 $\mu \mathrm{m}$ (11)) bioink was prepared by dispersing first HAp in the aforementioned buffer after which gellan gum and alginate were added. The solution was then allowed to dissolve with stirring at $90^{\circ} \mathrm{C}$ for $1 \mathrm{~h}$. Afterwards, the solution was transferred into a beaker and continuously mixed with a spatula until cooled to RT to avoid the formation of a gel-block due to the thermal gelation of gellan gum. The obtained paste was loaded into a double syringe (L-system, Medmix) and mixed 10:1 with media (DMEM 31966, Gibco) through a static mixer (MLX 2.5-16-LLM, Medmix), filled into a printer cartridge and centrifuged at $1500 \mathrm{rcf}$ for $4 \mathrm{~min}$ to remove any remaining air bubbles. Four different alginate-gellan gum bioinks were used. Bioink 1: 3\% GG, 2\% Alg, Bioink 2: 3\% GG, 2\% Alg, Bioink 3: 4\% GG, 2\% Alg $_{K}$, Bioink 4: 3\% GG, 2\% Alg, 5\% HAp (Figure 1 C).

Additionally, two gelatin-based bioinks were prepared. $(25,26)$ A gelatin-alginate bioink was prepared by dissolving gelatin (7\%, type A, porcine, 300 bloom, sigma) and alginate (1\%, Algin I-1, Kimica) in $220 \mathrm{mM}$ d-glucose and $20 \mathrm{mM}$ HEPES, $\mathrm{pH} 7.4$, at $60^{\circ} \mathrm{C}$ for $1 \mathrm{~h}$ while stirring (gelatin bioink). A GelMA-Laponite bioink was prepared by first dispersing Laponite (1\%, LaponiteRD, BYK Additives \& Instruments) in $220 \mathrm{mM}$ d-glucose and $20 \mathrm{mM}$ HEPES, $\mathrm{pH} 7.4$, under stirring and then 
A

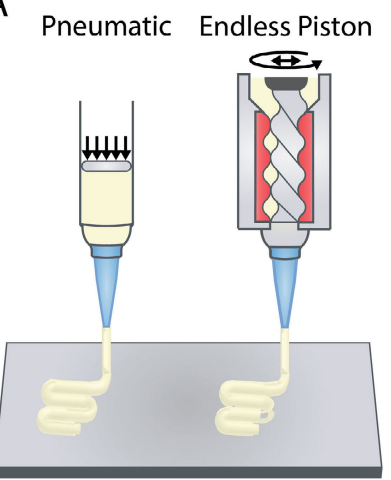

B

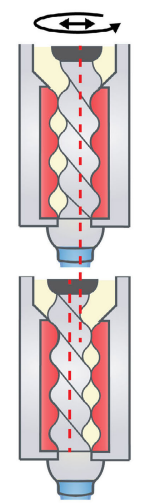

\begin{tabular}{|c|c|}
\hline ramemac & Gellan Gum (GG) \\
\hline & Alginate $_{\text {Pronova }}\left(\mathrm{Alg}_{\mathrm{p}}\right)$ \\
\hline & Alginate $_{\text {Kimica }}\left(\mathrm{Alg}_{\mathrm{K}}\right)$ \\
\hline - & Hydroxyapatite (HAp) \\
\hline 3 & Gelatin \\
\hline $\tan ^{2}$ & GelMA \\
\hline 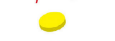 & Laponite \\
\hline & Bovine Chondrocyte \\
\hline
\end{tabular}

C

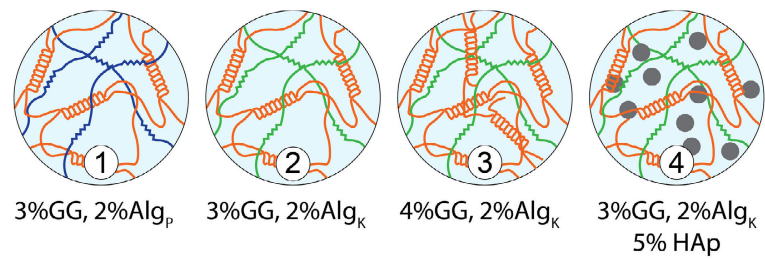

E

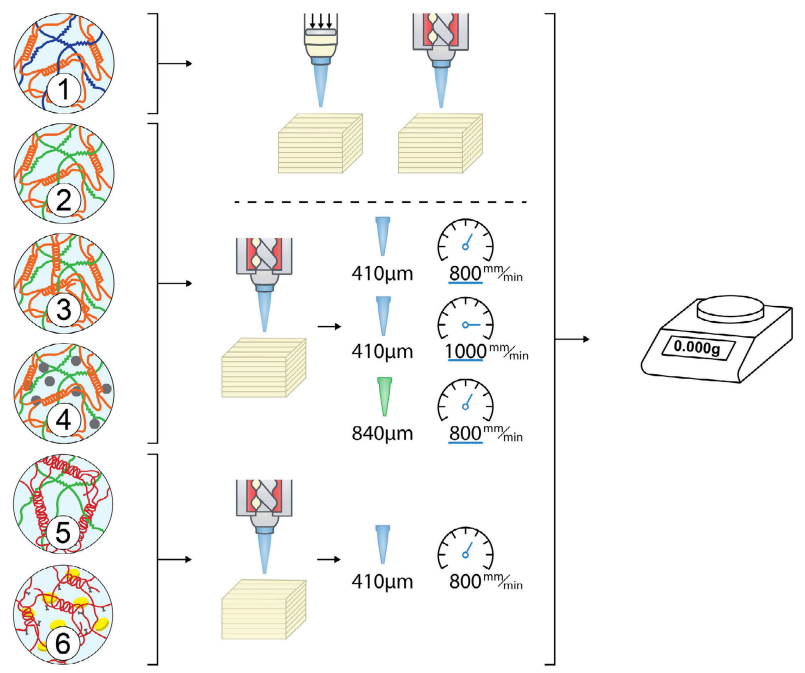

$\mathrm{F}$
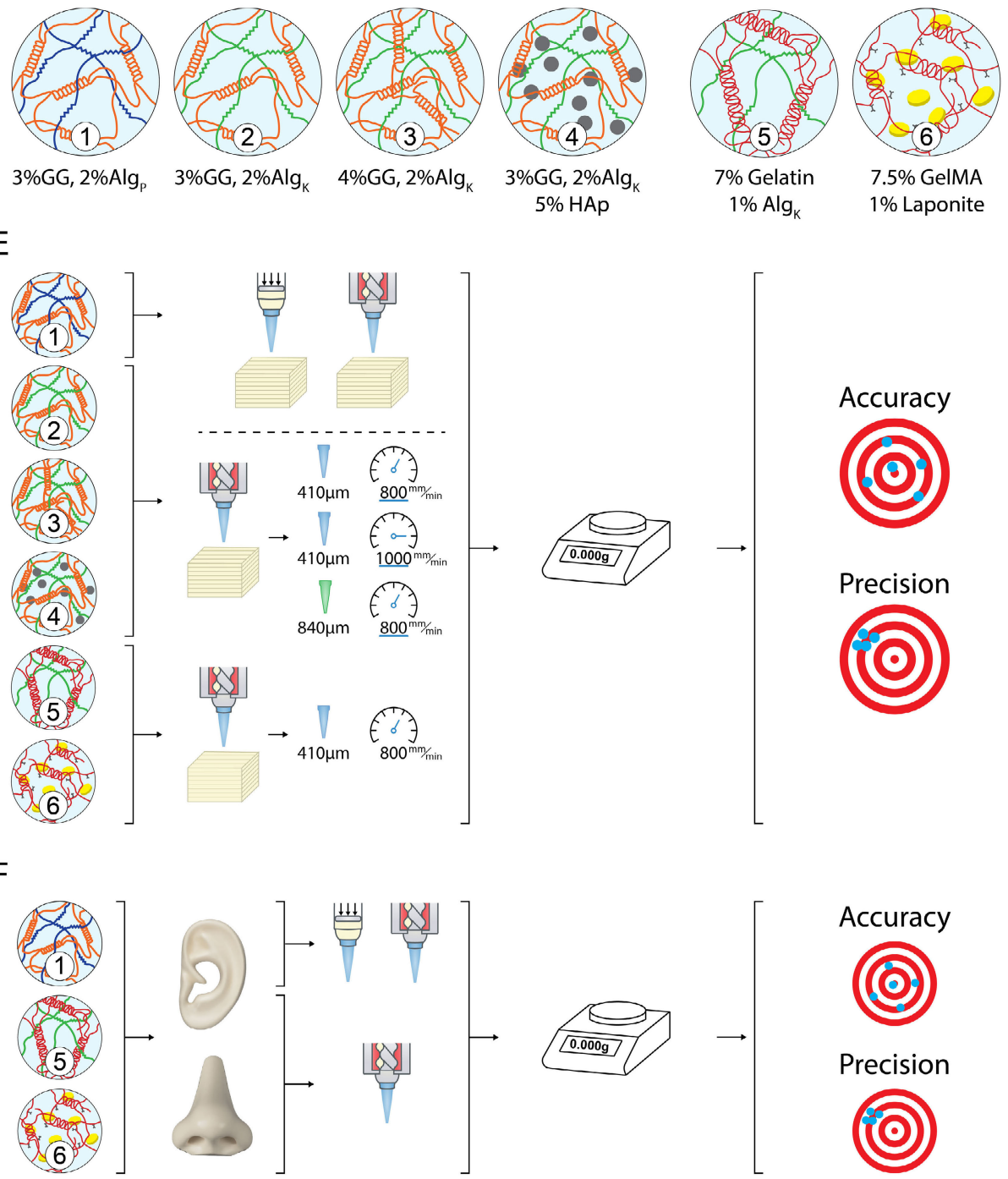

7.5\% GelMA

$1 \%$ Laponite

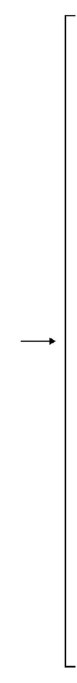

Accuracy

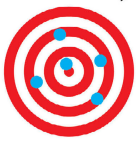

Precision

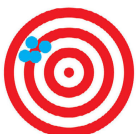

Accuracy

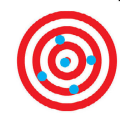

Precision

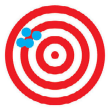

G

Viability

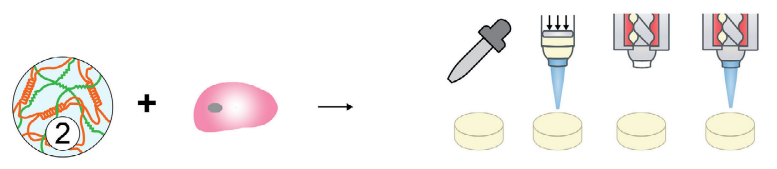<smiles>CC1CC1</smiles>

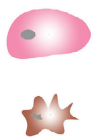

Figure 1 A, Illustration of the two different extrusion modes compared in this study. B, Sequential images of material being pushed through a PCP while the rotor is moving eccentrically (red dotted line). Light-yellow regions are cavities filled with bioink. C, Schematic of the different alginate/gellan gum bioinks (1-4) and $\mathbf{D}$, the gelatin-based bioinks (5-6) used in this study. $\mathbf{E}$, Schematic of the evaluation process. The bioprinting process for Bioink 1 was first optimized for both pneumatic and PCP extrusion. Afterwards, bioprinted constructs were weighed, and the accuracy and precision of the two systems compared. Bioinks $2-4$ were used to evaluate the precision and accuracy of the PCP with different printing parameters: constructs were printed with a $410 \mu \mathrm{m}$ nozzle at $800 \mathrm{~mm} / \mathrm{min}, 410 \mu \mathrm{m}$ nozzle at $1000 \mathrm{~mm} / \mathrm{min}$ and $840 \mu \mathrm{m}$ nozzle at $800 \mathrm{~mm} / \mathrm{min}$. F, Bioprinting of complex structures such as the ear and nose. Bioink 1 was used to compare the pneumatic extrusion to the PCP extrusion whereas the thermogelling bioinks, Bioink 5 and 6 , were printed with the PCP alone and evaluated for accuracy and precision. G, Bioprinting of Bioink 2 with 3 and 10 million bovine chondrocytes/ml. Samples were cast or bioprinted with the pneumatic system with a $410 \mu \mathrm{m}$ nozzle and the PCP with and without a $410 \mu \mathrm{m}$ nozzle. Viability was assessed immediately, one and 14 days after bioprinting. 
1 adding GelMA (7.5\%) which was allowed to dissolve at $60^{\circ} \mathrm{C}$ for $3 \mathrm{~h}$ under stirring (GelMA bioink). Subsequently, both gelatin-based bioinks were filled into printing cartridges and cooled to $37^{\circ} \mathrm{C}$. Before printing, the cartridges were kept at room temperature for $20 \mathrm{~min}$ and then loaded into the bioprinter.

\subsection{Rheology}

Rheological characterization of the bioinks was performed on an Anton Paar MCR 301 rheometer equipped with a peltier element and thermal hood (H-PTD 200; Anton Paar, Switzerland). Before each measurement, the respective geometries were coated with poly-L-lysine (PLL) to prevent slippage between the material and the geometry. A $10 \mu \mathrm{g} / \mathrm{ml}$ PLL solution was placed on the plate of the rheometer and the geometry brought in close contact with the solution so that the solution was fully covering the area of the geometry. The temperature was raised to $37^{\circ} \mathrm{C}$ for $30 \mathrm{~min}$ after which the solution was removed and the bioink added. Three samples per bioink batch were tested and three batches of each bioink were prepared. Tests were performed at $25^{\circ} \mathrm{C}$ unless otherwise stated.

Shear thinning behavior was characterized by performing rotational tests with a $20 \mathrm{~mm}$ parallel plate geometry (PP20, Anton Paar) at a gap of $0.8 \mathrm{~mm}$. The shear rate was increased logarithmically from $0.01 \mathrm{~s}^{-1}$ to $300 \mathrm{~s}^{-1}$.

Shear recovery behavior was characterized by performing oscillatory tests with the same setup as in shear thinning tests. The test was split into several intervals with alternating shear strain, constant frequency of $1 \mathrm{~Hz}$ and measurement point duration of $10 \mathrm{~s}$. In the first interval, a strain of $1 \%$ was applied for 300 s, i.e. 30 measurement points of $10 \mathrm{~s}$ each, after which an interval of high shear strain was applied at $500 \%$ for 60 s. A short resting phase of $5 \mathrm{~s}$ followed to allow the material to come to rest after which the recovery of the material was measured at $1 \%$ shear strain for $900 \mathrm{~s}$. The high shear strain, $5 \mathrm{~s}$ rest and recovery interval were then repeated once.

Stress amplitude sweep tests were performed in oscillation with the same setup as in shear thinning tests. Shear stress was increased logarithmically from $0.5 \mathrm{~Pa}$ to $25 \mathrm{~Pa}$ at a frequency of $1 \mathrm{~Hz}$. Yield point was calculated based on the "Yield Stress II" analysis in the Rheoplus software (Anton Paar). A tolerated deviation of 5\% from the plateau stress value of the linear viscoelastic range was set as the threshold to determine the yield point.

Crosslinking was characterized by performing oscillatory measurements utilizing a $10 \mathrm{~mm}$ parallel plate geometry (PP10, Anton Paar) at a gap of $0.8 \mathrm{~mm}$. Tests were performed at $0.1 \%$ shear strain and $1 \mathrm{~Hz}$. For the alginate-gellan gum bioink, a measurement point duration of $20 \mathrm{~s}$ was chosen. After $5 \mathrm{~min}, 1 \mathrm{ml}$ of $20 \mathrm{mM} \mathrm{CaCl}_{2}$ was added around the geometry to allow diffusion of calcium ions into the bioink and initiate crosslinking. The gelatin-alginate bioink was tested at $37^{\circ} \mathrm{C}$ at a measurement point duration of $10 \mathrm{~s}$. The temperature was increased to $37^{\circ} \mathrm{C}$ to simulate culture conditions in which gelatin would liquify and the stiffness of the gel depend on the crosslinked alginate network. After $1 \mathrm{~min}, 1 \mathrm{ml}$ of $100 \mathrm{mM} \mathrm{CaCl}_{2}$ was added around the geometry and the gel allowed to crosslink for $1 \mathrm{~h}$. Similarly, the GelMALPN bioink was tested at $37^{\circ} \mathrm{C}$ and $10 \mathrm{~s}$ per measurement point. The GelMA-LPN bioink was combined with lithium phenyl-2,4,6-trimethylbenzoylphosphinate (LAP) to reach a final concentration of $0.1 \%$ LAP. After $5 \mathrm{~min}$, crosslinking was initiated by UV exposure (OmnicureSeries 1000, LumenDynamics, 320-500 nm wavelength, $9.55 \mathrm{~mW} / \mathrm{cm} 2$ ) for $5 \mathrm{~min}$ and the crosslinked gel tested for another $10 \mathrm{~min}$.

\subsection{Preparation process of the pneumatic system and the progressive cavity pump}

To reliably compare the two printing systems, several key parameters influencing the material deposition during bioprinting, were analyzed. These parameters included 1) die swell behavior after extrusion, 2) volume flow of the bioink at different pressures, 3) extrusion delay of the individual systems before material extrusion and 4) layer height of up to 3 consecutive layers. All tests were performed with Bioink 1, where three batches of bioink were prepared for each test and each test repeated three times. Imaging was performed on a stereomicroscope (Leica Wild M650) equipped with a color camera (Leica EC3) and all images analyzed in ImageJ. To relate the extruded weight to the extruded volume, density was calculated by extruding $200 \mu \mathrm{l}$ of bioink with a positive displacement pipette (microman, Gilson) into a test tube and weighing the material. All printing related to the comparison between pneumatic system and PCP was performed with a $410 \mu \mathrm{m}$ nozzle. Previous studies showed that such a nozzle diameter provides a good compromise between cell viability and good resolution. (23, 24) Additional information regarding each test can be found in the Supplementary Methods.

1) Die swell: To measure the strand diameter after extrusion, the cartridge connected to the pneumatic system and the PCP were mounted on a stand allowing the free vertical extrusion of the bioink. Images of the extruded strand during flow were then taken at the nozzle tip and analyzed.

2) Volume flow of the pneumatic system: Volume flow of the pneumatic system was measured at various pressures by connecting the printing cartridge to the 
1 bioprinter and extruding bioink for $30 \mathrm{~s}$ into test tubes after which the extruded amount was determined and the volume flow calculated.

3) Extrusion delay: To assess the extrusion delay, first line height and width of printed strands were measured. Individual lines were printed onto glass slides using different pressures for the pneumatic system and different volume flow rates for the PCP and imaged from the top and side. Line height and width were then analyzed in ImageJ. These data were used to adjust the nozzle distance to the collecting glass slide. The extrusion delay was assessed by creating a $15 \mathrm{~mm}$ long line in BioCAD and a delay of 0,100, 200, 300 and 400 ms was incorporated using the G4 function (interrupt program execution) right after the extrusion command. Lines were printed with different delays after which the printed line was imaged and its length analyzed in ImageJ. The optimal delay was then calculated based on the delay at which the printed line achieved the length of the line designed in BioCAD.

4) Layer height: Bioprinting layers of material is not only dependent on the shear recovery behavior of the bioink but also on the fusion of strands deposited next and on top of each other. (24) To assess the height of the resulting layers, 10 lines were printed next to each other, spaced $410 \mu \mathrm{m}$ apart.

We determined the ideal volume flow assuming a square cross section of the strand with side length of the square equal to the nozzle diameter. As strands fuse, any cavities are filled and surface tension evens the surface of the printed construct. The volume flow can thus be determined as follows:

$$
Q=f \cdot a^{2}
$$

with $f$ being the feed rate $(\mathrm{mm} / \mathrm{min})$ and $a$ being the diameter of the printing nozzle. Applying this equation to a $410 \mu \mathrm{m}$ nozzle and a feed rate of $800 \mathrm{~mm} / \mathrm{min}$ the volume flow was determined to be $134.5 \mu \mathrm{l} / \mathrm{min}$. Regarding the PCP, this volume flow can be precisely set in the control unit whereas for the pneumatic system, the pressure to achieve this volume flow $(25.33 \pm 0.37 \mathrm{kPa})$ was determined as described in 2). As the pressure sensors installed in the Biofactory had a resolution of $1 \mathrm{kPa}$ which did not allow a more precise adjustment, $26 \mathrm{kPa}$ were used to print with the pneumatic system leading to a volume flow of $145.8 \pm 9.0 \mu \mathrm{l} / \mathrm{min}$. Due to the unknown layer height, a layer height of $410 \mu \mathrm{m}$ was set to avoid collision of the printing nozzle with the extruded material. Up to three consecutive layers were printed on top of each other and printed constructs analyzed by imaging sideways and assessing the layer height in ImageJ.

$50 \quad 2.7$ Comparison of the accuracy and precision of

51 the pneumatic system to the progressive cavity 52 pump
Data from the preparation process were then used to design and print a test cube to compare the accuracy and precision of the pneumatic system to the PCP. The cube designed in BioCAD consisted of 26 lines spaced $410 \mu \mathrm{m}$ apart at a length of $10.25 \mathrm{~mm}$ which were connected alternately on either side, therefore creating one continuous printing path per layer. The designed layer was then stacked 14 times with a distance between each consecutive layer of $410 \mu \mathrm{m}$. The printing path was alternately rotated by $90^{\circ}$ on each consecutive layer. Cubes were printed at $800 \mathrm{~mm} / \mathrm{min}$ (Supplementary Figure $4 \mathrm{~A}$ ). A printing delay after the start command for extrusion of $155 \mathrm{~ms}$ for the pneumatic system and $284 \mathrm{~ms}$ for the PCP was included according to the pre-determined extrusion delay. Additionally, a layer height of $380 \mu \mathrm{m}$ and $403 \mu \mathrm{m}$ was used for the pneumatic system and the PCP respectively. As described previously when determining the printing pressure for the layer height analysis, $26 \mathrm{kPa}$ were used for the pneumatic system to achieve the corresponding volume flow. After printing, the weight of each cube was immediately measured. For all tests, three batches of Bioink 1 were used, and three cubes printed with each batch and each system.

The printing time of 3D printers and bioprinters are influenced by the acceleration and jerk (rate of change of acceleration) of the systems. (34) Therefore it is not possible to simply determine the print-time based on the length of the printing path and the feed rate, i.e. the set velocity at which the printing nozzle moves, without knowing the specifications of the individual system. This means for example, that a $10 \mathrm{~mm}$ line printed with a feed rate of $10 \mathrm{~mm} / \mathrm{s}$ does not require $1 \mathrm{~s}$ to be printed. As the printer needs to accelerate to achieve the feed rate (print velocity) and decelerate to come to a stop, the actual print takes longer than $1 \mathrm{~s}$. Additionally, at corners, the printer needs to decelerate e.g. in x-direction and accelerate in ydirection, meaning that the movement comes to a momentary stop which can lead to increased material deposition at these positions. (34) To determine the exact volume extruded during the print process, the print-time, i.e. the time between the start and stop signal of the g-code was measured and the targeted volume calculated according to:

$$
V=t \cdot \dot{Q}
$$

with $\mathrm{V}$ being the targeted volume, $\mathrm{t}$ being the time the pressure valve is open and $\dot{Q}$ being the set volume flow. For the cubes described in this section, the print time was $333.9 \mathrm{~s}$ and with a volume flow of $134.5 \mu \mathrm{l} / \mathrm{min}$, the targeted volume was calculated to be $748.5 \mu$ l for the PCP and with a volume flow of $145.8 \mu \mathrm{l} / \mathrm{min}$, the targeted volume was $811.4 \mu \mathrm{l}$ for the pneumatic system.

Accuracy was determined based on the percentage difference between the mean of the measured extruded 
1 volume and the targeted volume and precision determined based on the standard error.

Microscopic analysis of printed strands of Bioink 1 was further performed to evaluate differences in the extrusion behavior. Individual strands were printed on glass slides and their width analyzed. Three different batches of bioink were evaluated with each system. With each batch, three different parts of the bioprinting cartridge were printed and with each part, three samples were printed. Part 1 corresponded to the initial portion of the cartridge, while part 2 and part 3 corresponded to the middle and last portion of the cartridge. Printed lines were imaged with a stereomicroscope (Leica Wild M650).

\subsection{Influence of the bioink composition, printing speed and nozzle diameter on the accuracy and precision of the progressive cavity pump}

To compare the influence of different printing parameters and different bioinks on the precision and accuracy of the PCP, cubes were printed with different nozzle sizes and different feed rates and each test carried out with Bioinks 2-6 (Figure $1 \mathrm{E}$ ). Cubes as described in Supplementary Figure $4 \mathrm{~A}$, designed for a $410 \mu \mathrm{m}$ nozzle were printed at $800 \mathrm{~mm} / \mathrm{min}$ and $1000 \mathrm{~mm} / \mathrm{min}$. Additionally, cubes designed for a nozzle of twice the diameter, i.e. $840 \mu \mathrm{m}$, were printed at $800 \mathrm{~mm} / \mathrm{min}$ to demonstrate the required volume flow adjustment when increasing nozzle diameter. These cubes consisted of 14 lines spaced $840 \mu \mathrm{m}$ apart at a length of $10.92 \mathrm{~mm}$ in a similar arrangement as in the cubes printed with a $410 \mu \mathrm{m}$ nozzle. A total of 8 layers were printed (Supplementary Figure 4 B). All cubes were printed three times with each bioink batch and three batches of bioink were prepared for each test. All tests were carried out by calculating the desired volume flow according to Equation (1) (Supplementary Table 1). The expected volume for each cube based on Equation 2 was therefore: $748.49 \mu \mathrm{l}$ for cubes printed with a $410 \mu \mathrm{m}$ nozzle at $800 \mathrm{~mm} / \mathrm{min}$, $763.48 \mu \mathrm{l}$ for cubes printed with a $410 \mu \mathrm{m}$ nozzle at 1000 $\mathrm{mm} / \mathrm{min}$ and $1034.63 \mu \mathrm{l}$ for cubes printed with a $840 \mu \mathrm{m}$ nozzle at $800 \mathrm{~mm} / \mathrm{min}$.

The tests described in this section were carried out without performing the printing preparation as described in Section 2.6. A delay of $284 \mathrm{~ms}$ was used for all tests as it was assumed that the delay arises from the signaling between bioprinter, control unit of the PCP and PCP itself as well as the start of the rotor of the PCP and not due to nozzle size or material used. The layer height was set to the nozzle diameter as Equation (1) ideally assumes a printed line with square cross-sectional area of side length of the nozzle diameter.

\subsection{Printing of thermosetting bioinks}

Similar to Section 2.7, cubes were printed with the gelatin based bioinks with the PCP. Before printing, both bioinks were kept at $37^{\circ} \mathrm{C}$ and then allowed to cool at room temperature for $20 \mathrm{~min}$. Cubes designed for a 410 $\mu \mathrm{m}$ nozzle were printed at $800 \mathrm{~mm} / \mathrm{min}$ with a volume flow of $134.5 \mu \mathrm{l} / \mathrm{min}$. Three batches of each bioink were prepared and three cubes per bioink were printed. The expected volume for these cubes according to Equation (2) was $748.49 \mu \mathrm{l}$. These tests were again carried out without performing the printing preparation and a delay of 284 ms was used.

\subsection{Printing of complex structures}

While the designed cubes run on a continuous printing path with linear movements per layer, 3D printing and bioprinting procedures are generally more complex. Such print jobs commonly include printing of perimeter and infill leading to intermittent dispensing. We therefore printed ears and noses as sample structures with complex printing paths.

To record the extrusion process of these structures and thereby calculate the print-time, we recorded the on/off signal of the bioprinter using an Arduino combined with a voltage regulator. Similar to Section 2.6, knowing the volume flow, the time of dispensing allowed us to calculate the expected weight of the construct after the printing process and compare it to the actual weight. A volume of $1484.70 \mu \mathrm{l}$ was determined for the ear and $1526.96 \mu \mathrm{l}$ for the nose. Three batches of bioink were prepared and one ear and one nose printed per batch of bioink. Bioink 2 was used to print ears and noses with the pneumatic system and the PCP, whereas the gelatin-based bioinks were only printed with the PCP as pressure adjustments would have been necessary for the pneumatic system to ensure continuous volume flow. For the pneumatic system, $26 \mathrm{kPa}$ were used together with a delay of $155 \mathrm{~ms}$ and a volume flow of $134.5 \mu \mathrm{l} / \mathrm{min}$ and a delay of $284 \mathrm{~ms}$ was used for the PCP. All structures were printed with a $410 \mu \mathrm{m}$ nozzle and a feedrate of 800 $\mathrm{mm} / \mathrm{min}$.

\subsection{Cell Culture \& Viability}

Bovine articular chondrocytes were harvested as reported previously. (23) Briefly, full thickness articular cartilage was harvested from the medial and lateral femoral condyles of $\sim 6$ months old calves obtained from the local slaughterhouse. The cartilage was minced into pieces and digested in $0.03 \%$ collagenase in DMEM supplemented with $1 \%$ penicillin-streptomycin (Gibco). The solution was then filtered through a $40 \mu \mathrm{m}$ cell 
strainer and cells collected by centrifugation. Cells were plated at 10 '000 cells $/ \mathrm{cm}^{2}$ for the first passage and collected at $80 \%$ confluency by trypsinization. For further passaging, cells were expanded by seeding 3'000 cells $/ \mathrm{cm}^{2}$ and cultured up to the desired passage. Culturing was performed in DMEM (31966, Gibco) supplemented with $10 \%$ FBS, $50 \mu \mathrm{g} / \mathrm{ml}$ L-ascorbate-2-phosphate and 10 $\mu \mathrm{g} / \mathrm{ml}$ gentamicin at $37^{\circ} \mathrm{C}, 5 \% \mathrm{CO}_{2}$ and $95 \%$ humidity

At confluency cells were collected, resuspended at the desired concentration and combined with the bioink (Section 2.3) using a 10:1 double syringe (bioink: cell solution) and a static mixer element (MLX 2.5-16-LLM, Medmix). Two conditions were tested, either 3\% GG, 2\% Alg $_{K}$ and 3 million bovine chondrocytes per ml or 3\% GG, 2\% Alg $_{K}$ and 10 million bovine chondrocytes per ml. Cylindrical samples of $6 \mathrm{~mm}$ diameter and $1 \mathrm{~mm}$ height were either cast or printed with the pneumatic system or the PCP, both with a $410 \mu \mathrm{m}$ nozzle. To evaluate whether the stator/rotor of the PCP would damage cells or the extrusion through the nozzle, additional samples were prepared with bioink only extruded through the stator/rotor and bioink extruded through the stator/rotor and a nozzle (Figure $1 \mathrm{G}$ ). Samples were cultured in chondrogenic medium composed of DMEM (31966, Gibco) supplemented with $50 \mu \mathrm{g} / \mathrm{ml}$ L-ascorbate-2phosphate, $40 \mu \mathrm{g} / \mathrm{ml}$ L-proline, 1\% AntibioticAntimycotic (Gibco), 1\% ITS+ (ITS+ Premix Universal Culture Supplement, Corning) and $10 \mathrm{ng} / \mathrm{ml}$ TGF- $ß 3$ (Recombinant Human TGF-ß3, PeproTech) at $37^{\circ} \mathrm{C}, 5 \%$ $\mathrm{CO}_{2}$ and $95 \%$ humidity. A total of three samples was tested per timepoint and each sample imaged at three different locations.

Samples were printed, crosslinked in $100 \mathrm{mM} \mathrm{CaCl}_{2}$ and immediately stained for viability (day 0). Further, viability was assessed one day after printing for the 3 million cells/ml condition and one and 14 days after printing for the 10 million cells/ml condition. Samples were washed three times in media (DMEM 31966, Gibco), stained in $1 \mathrm{uM}$ CalceinAM and $1 \mathrm{uM}$ propidium iodide in media (DMEM 31966, Gibco) for $1 \mathrm{~h}$ and washed again three times in media (DMEM 31966, Gibco). Samples were imaged on a structured illumination microscope (Zeiss Axio Observer equipped with an Apotome) from the surface of the sample $100 \mu \mathrm{m}$ into the sample with images being acquired every $5 \mu \mathrm{m}$ for day 0 and day 1. Due to the proliferation of cells, a 2-photon microscope (Leica SP-8 multiphoton) was used at day 14 to be able to image $100 \mu \mathrm{m}$ into the sample. CalceinAM excitation was carried out at $900 \mathrm{~nm}$ and propidium iodide excitation was carried out at $1100 \mathrm{~nm}$. Images were acquired every $1 \mu \mathrm{m}$. Stacks were projected up to $50 \mu \mathrm{m}$ onto the z-plane and viability calculated by dividing the number of viable cells by the number of total cells.

\subsection{Histological and immunohistological analysis}

Histological and immunohistological analysis was performed on samples casted or printed with 10 million bovine chondrocytes per $\mathrm{ml}$ bioink. Samples were cultured in chondrogenic medium at $37^{\circ} \mathrm{C}, 5 \% \mathrm{CO}_{2}$ and $95 \%$ humidity for 4 weeks after which samples were fixed in $4 \%$ paraformaldehyde for $4 \mathrm{~h}$. Samples were then dehydrated in graded ethanol solutions. $5 \mu \mathrm{m}$ sections were cut on a microtome and sections were deparaffinized and rehydrated before the stainings. Brightfield imaging was performed on an automated slide scanner (Panoramic 250, 3D Histech). Three samples per condition were stained. As positive control, bovine articular cartilage (Supplementary Figure 6) was stained.

Safranin O staining was performed by staining sections in Weigert's Iron Hematoxylin solution for 5 min after which sections were washed in deionized water. Following, sections were differentiated in $1 \%$ acidalcohol (1\% $\mathrm{HCl}$ in $70 \%$ ethanol) for $2 \mathrm{~s}$, washed and stained in $0.02 \%$ Fast Green for $1 \mathrm{~min}$. Sections were destained in $1 \%$ acetic acid and stained in $1 \%$ Safranin O for $30 \mathrm{~min}$. Afterwards, sections were dehydrated to xylene and mounted.

Immunohisotogical stainings were performed by first retrieving the antigen with hyaluronidase solution at $37^{\circ} \mathrm{C}$ for $30 \mathrm{~min}$. Sections were washed in PBS and then blocked in 5\% bovine serum albumin (BSA) for $1 \mathrm{~h}$. Afterwards the primary antibody (collagen I: abcam 138492, rabbit anti collagen I, diluted 1:1500 and collagen II: DSHB Hybridome Product II-II6B3, deposited by Linsenmayer, T.F., diluted 1:20) diluted in 1\% BSA was applied at $4^{\circ} \mathrm{C}$ overnight. Sections were washed in PBS the following day, incubated in $0.3 \% \mathrm{H}_{2} \mathrm{O}_{2}$ for $15 \mathrm{~min}$, washed again in PBS and incubated with the secondary antibody (goat anti-rabbit IgG-HRP, ab6721, abcam or goat anti-mouse IgG-HRP, ab6789, abcam, diluted $1: 1000)$ at room temperature for $1 \mathrm{~h}$. Sections were then washed in PBS and developed with the DAB substrate kit (ab64238, abcam) according to the manufacturer specifications for $5 \mathrm{~min}$. Afterwards, sections were washed in deionized water, stained in Weigert's Iron Hematoxylin for $3 \mathrm{~min}$, washed, destained in 1\% acid alcohol for $2 \mathrm{~s}$ and washed again. Bluing was performed in $0.1 \%$ sodium carbonate for $1 \mathrm{~min}$, sections briefly washed, dehydrated to xylene and mounted.

\subsection{Statistical analysis}

All statistical analyses were carried out in Matlab (Matlab 2018a, Mathworks). One- and two-way analyses of variance (ANOVA) were performed followed by Bonferroni multiple comparisons. A p-value below 0.05 was considered statistically significant $\left({ }^{*} \mathrm{p}<0.05\right.$, ${ }^{* *} \mathrm{p}<$ 
$0.005, * * * \mathrm{p}<0.001)$. Accuracy and precision testing was performed printing three samples per bioink batch and three different batches of each bioink were used, resulting in a total of nine samples. Viability was analyzed imaging three samples per timepoint at three different locations of the sample. Reported values represent mean \pm standard deviation (SD).

\section{Results}

\subsection{PCP installation}

To confirm the ability of the PCP to deposit a precise volume at different volume flows, we extruded a specific amount of material and weighed it. These tests were necessary to ensure proper flow rates for the following experiments as the calibration of the PCP was carried out at a higher volumetric flow rate $(370 \mu \mathrm{l} / \mathrm{min})$ than used in this study. Additionally some experiments were conducted below the minimum volumetric flow rate of the PCP $(120 \mu \mathrm{l} / \mathrm{min})$ as listed in its technical specifications. For a desired $50 \mathrm{mg}$ of material, the PCP extruded 49.84 $\pm 0.27 \mathrm{mg}$ at $100 \mu \mathrm{l} / \mathrm{min}, 49.88 \pm 0.30 \mathrm{mg}$ at $150 \mu \mathrm{l} / \mathrm{min}$ and $49.92 \pm 0.13 \mathrm{mg}$ at $200 \mu \mathrm{l} / \mathrm{min}$, which did not show a significant dependency on the volume flow (Supplementary Figure 3 D, n.s.).

\subsection{Rheological characterization}

All alginate-gellan gum bioinks used in this study showed the characteristic shear recovery and shear thinning behavior with yield point necessary to achieve good printability (Table 1, Supplementary Figure 2 A-L). To determine if the various bioinks differed in their rheological behavior, which ultimately determines the bioinks' printing pressure and layer-by-layer deposition, key rheological parameters were compared. These parameters provide additional information on the homogeneity of the different bioink batches based on the batch's standard deviation and on the reproducibility of the bioink preparation based on the difference in these values between batches. Viscosity was analyzed at the initial shear rate of $0.01 \mathrm{~s}^{-1}\left(\eta_{0.01}\right)$, yield stresses $\left(\tau_{\mathrm{y}}\right)$ of the different bioinks compared and the shear recovery behavior evaluated based on the storage modulus ( $G^{\prime}$ ) recovery and percentage recovery of G'.

A significant increase in viscosity was observed when switching from $\mathrm{Alg}_{\mathrm{P}}$ to $\mathrm{Alg}_{\mathrm{K}}\left({ }^{*} \mathrm{p}<0.05\right)$. When increasing the GG concentration from $3 \%$ to $4 \%$, the additional polymer content led to an increase in viscosity (***p < 0.001). Similarly, when incorporating hydroxyapatite microparticles into the bioink, additional frictional forces occur, (35) leading to an increase in viscosity $\left({ }^{* * *} \mathrm{p}<\right.$ 0.001, Table 1, Supplementary Figure 2 P).

Yield stress was higher for the bioink prepared with Algp compared to the one prepared with $\operatorname{Alg}_{K}(* * * p<$ 0.001). Contrary to the viscosity analysis, no significant difference was observed in the yield stress when increasing the GG concentration from $3 \%$ to $4 \%$ (n.s.), while the addition of HAp increased the yield stress $\left({ }^{* * *} \mathrm{p}\right.$ $<0.001$, Table 1, Supplementary Figure 2 Q).

Analysis of the shear recovery behavior of the bioinks was carried out based on their storage moduli (G'), which in combination with the loss modulus ( $G$ '”) provides information on the differences in the viscoelastic behavior of the bioinks. (35) No significant difference in the initial $G^{\prime}\left(G_{i}^{\prime}\right)$ as well as the $G^{\prime}$ after the first $\left(G_{1}^{\prime}\right)$ and second $\left(G_{2}^{\prime}\right)$ shear event occurred when changing the alginate origin from $A \lg _{\mathrm{P}}$ to $\operatorname{Alg}_{\mathrm{K}}\left(\mathrm{p}_{\mathrm{i}}=\right.$ n.s., $\mathrm{p}_{1}=$ n.s., $\mathrm{p}_{2}=$ n.s.) whereas Bioink 2 showed a significantly lower G', before shearing but not after the first and second shear event $\left({ }^{*} \mathrm{p}_{\mathrm{i}}\right.$ $<0.05, \mathrm{p}_{1}=$ n.s., $\mathrm{p}_{2}=$ n.s.). Increasing the GG concentration significantly increased all storage moduli $\left({ }^{* * *} \mathrm{p}_{\mathrm{i}}<0.001,{ }^{* * *} \mathrm{p}_{1}<0.001,{ }^{* * *} \mathrm{p}_{2}<0.001\right)$ as well as loss moduli $\left(* * * \mathrm{p}_{\mathrm{i}}<0.001,{ }^{* * *} \mathrm{p}_{1}<0.001,{ }^{* * *} \mathrm{p}_{2}=0.001\right)$. Similarly, the addition of HAp to Bioink 2 increased both G' $\left({ }^{*} \mathrm{p}_{\mathrm{i}}<0.05,{ }^{*} \mathrm{p}_{1}<0.05,{ }^{*} \mathrm{p}_{2}<0.05\right)$ and G' $\left({ }^{* * *} \mathrm{p}_{\mathrm{i}}<\right.$ $\left.0.001, * * * \mathrm{p}_{1}<0.001,{ }^{* * *} \mathrm{p}_{2}<0.001\right)$ significantly but did not increase it as strong as the increase in GG concentration (Table 1, Supplementary Figure 2 R). Despite these differences, no significant differences were found in the percentage shear recovery of G' after the first shear event (Bioink 1: $79.7 \pm 4.1 \%$, Bioink 2: $75.5 \pm 1.0 \%$, Bioink 3: $74.6 \pm 2.2 \%$, Bioink 4: $76.8 \pm 2.2 \%)$ and only a significant difference between Bioink 1 and $3\left({ }^{*} \mathrm{p}<0.05\right)$ was found after the second shear event (Bioink 1: $78.3 \pm$

Table 1: Key rheological parameters determined for the various bioinks. Viscosity at the initial shear rate of $0.01 \mathrm{~s}^{-1}\left(\eta_{0.01}\right)$, yield stress $\left(\tau_{\mathrm{y}}\right)$, initial sorage modulus $\left(G^{\prime}{ }_{i}\right)$ and storage modulus after the first $\left(G^{\prime}{ }_{1}\right)$ and second $\left(G^{\prime}{ }_{2}\right)$ shear event and initial loss modulus $\left(G^{\prime \prime}{ }_{i}\right)$ and loss modulus after the first $\left(G^{\prime \prime}{ }_{1}\right)$ and second $\left(G^{\prime \prime}{ }_{2}\right)$ shear event. Mean $\pm S D, n=9$ (three batches).

\begin{tabular}{|c|c|c|c|c|c|c|c|c|}
\hline & $\eta_{0.01}[\mathrm{~Pa} \cdot \mathrm{s}]$ & $\tau_{\mathrm{y}}[\mathrm{Pa}]$ & $\mathrm{G}_{\mathrm{i}}^{\prime}[\mathrm{Pa}]$ & $\mathrm{G}^{\prime}{ }_{1}[\mathrm{~Pa}]$ & $\mathrm{G}_{2}{ }_{2}[\mathrm{~Pa}]$ & $\mathrm{G},{ }_{i}[\mathrm{~Pa}]$ & $\mathrm{G}^{\prime \prime}{ }_{1}[\mathrm{~Pa}]$ & $\mathrm{G},{ }_{2}[\mathrm{~Pa}]$ \\
\hline Bioink 1 & $1357 \pm 140$ & $6.72 \pm 0.40$ & $1179 \pm 255$ & $935 \pm 169$ & $917 \pm 162$ & $717 \pm 105$ & $312 \pm 24$ & $285 \pm 34$ \\
\hline Bioin & $1638 \pm 209$ & $5.29 \pm 0.86$ & $1295 \pm 107$ & $978 \pm 85$ & $957 \pm 81$ & $492 \pm 55$ & $338 \pm 34$ & $333 \pm 32$ \\
\hline Bioink 3 & $2337 \pm 319$ & $5.71 \pm 0.40$ & $2029 \pm 248$ & $1516 \pm 213$ & $1475 \pm 209$ & $1125 \pm 84$ & $713 \pm 72$ & $692 \pm 69$ \\
\hline Bioink 4 & $2870 \pm 434$ & $6.38 \pm 0.29$ & $1619 \pm 226$ & $1247 \pm 202$ & $1208 \pm 202$ & $652 \pm 96$ & $494 \pm 69$ & $479 \pm 65$ \\
\hline
\end{tabular}


1 4.4\%, Bioink 2: $73.9 \pm 0.9 \%$, Bioink 3: $72.5 \pm 2.1 \%$, Bioink 4: $74.4 \pm 2.4 \%$ ).

As bioinks need to remain stable in culture post printing to support tissue maturation, crosslinking of the bioinks was performed (Supplementary Figure $2 \mathrm{M}-\mathrm{O}$ ). Storage moduli of $268.5 \pm 26.5 \mathrm{kPa}, 33.1 \pm 3.7 \mathrm{kPa}$ and $1.9 \pm 0.8 \mathrm{kPa}$ and loss moduli of $14.9 \pm 0.7 \mathrm{kPa}, 2.9 \pm 0.4$ $\mathrm{kPa}$ and $0.01 \pm 0.005 \mathrm{kPa}$ were reached for the alginategellan gum (Bioink 1), the gelatin-alginate and the GelMA-LPN bioink respectively.

\subsection{Preparation process of the pneumatic system and the progressive cavity pump}

1) Die swell: Measurements of the strand diameter after extrusion through a $410 \mu \mathrm{m}$ nozzle with the pneumatic system did not show any significant dependence on the extrusion pressure except for material extruded at $15 \mathrm{kPa}$ ( $\mathrm{d}=428 \pm 10 \mu \mathrm{m}$ ). A significantly smaller diameter compared to material extruded at $25 \mathrm{kPa}(\mathrm{d}=439 \pm 9 \mu \mathrm{m}$, $\left.{ }^{*} \mathrm{p}<0.05\right)$ and at $30 \mathrm{kPa}\left(\mathrm{d}=441 \pm 7 \mu \mathrm{m},{ }^{*} \mathrm{p}<0.05\right)$ was observed (Supplementary Figure $3 \mathrm{~A}$ ). No significant differences were found in strand diameter when extruded with the PCP between different volume flows (Supplementary Figure 3 B, n.s.). The overall diameter of bioink extruded with the pneumatic system was 6\% (436 $\pm 9 \mu \mathrm{m})$ and with the PCP 5\% (431 $\pm 6 \mu \mathrm{m})$ larger than the nozzle diameter. As this increase in diameter can possibly be explained by velocity profile rearrangements and/or mass balance considerations (36), die swell was neglected in the further preparation process.

2) Volume flow of the pneumatic system: Increasing the pressure at which the bioink is extruded significantly increased the volume flow (Supplementary Figure $3 \mathrm{C}$ ). A pressure of $25.33 \pm 0.37 \mathrm{kPa}$ was determined to achieve a volume flow of $134.5 \mu \mathrm{l} / \mathrm{min}$, corresponding to an optimal print with a $410 \mu \mathrm{m}$ nozzle and a feed rate of 800 $\mathrm{mm} / \mathrm{min}$. Due to the resolution of the pressure sensor of the Biofactory ( $1 \mathrm{kPa}$ ), the pressure was rounded up to 26 $\mathrm{kPa}$ which would lead to a volume flow of $145.8 \mu \mathrm{l} / \mathrm{min}$.

3) Extrusion Delay: Line height and line width analysis were performed to optimize the distance of the nozzle to the glass slide. A significant increase in line height and line width was observed with increasing pressure or volume flow for the two systems respectively (Supplementary Figure $3 \mathrm{E}-\mathrm{H},{ }^{*} \mathrm{p}<0.05$ ). Importantly a line height of $242 \mu \mathrm{m}$ was determined for material being extruded at $26 \mathrm{kPa}$ with the pneumatic system and $226 \mu \mathrm{m}$ was determined for material being extruded at 134.5 $\mu \mathrm{l} / \mathrm{min}$ with the PCP to ensure proper contact of the printed strand with the print bed.

To determine the delay between start command of the g-code and the actual extrusion of material (extrusion delay), lines of $15 \mathrm{~mm}$ were printed at a height of $242 \mu \mathrm{m}$ and $226 \mu \mathrm{m}$ with the pneumatic system and PCP respectively. The delay was then calculated from lines printed at different delay times and data later interpolated to achieve a total line length of $15.41 \mathrm{~mm}$. The additional $0.41 \mathrm{~mm}$ were added as the distance from the center of the nozzle at the start point to the end point was set to $15 \mathrm{~mm}$ and therefore an overlap of half the nozzle diameter at the start and end had to be added. Accordingly, a delay of 155 ms and 284 ms was calculated for the pneumatic system and the PCP respectively (Supplementary Figure 3 I, J).

4) Layer Height: When printing lines next to each other, these lines fuse depending on the flow and viscoelastic behavior of the bioink and the overlap of the individual strands. (24) Significant differences were found between the layer height of one, two and three layers printed with the pneumatic system (layer 1: $441 \pm$ $46 \mu \mathrm{m}$, layer 2: $319 \pm 22 \mu \mathrm{m}$, layer 3: $379 \pm 49 \mu \mathrm{m}$, **p $<0.005)$ with an average total layer height of $380 \pm 75$ $\mu \mathrm{m}$. No differences in layer height were observed in layers printed with the PCP (layer 1: $393 \pm 13 \mu \mathrm{m}$, layer 2: 407 $\pm 41 \mu \mathrm{m}$, layer 3: $410 \pm 62 \mu \mathrm{m}$, n.s.) having an average total layer height of $403 \pm 35 \mu \mathrm{m}$ (Supplementary Figure $3 \mathrm{~K}, \mathrm{~L}$ ). These results indicate a better extrusion control of the PCP compared to the pneumatic system.

\subsection{Comparison of the accuracy and precision of} the pneumatic system to the progressive cavity pump

Cubes designed based on the results of the preparation process were printed to compare the accuracy and precision of the pneumatic system to the PCP. Overall the PCP achieved a 41 times higher accuracy $(* * * \mathrm{p}<0.001)$ and 35 times higher precision $\left({ }^{* *} \mathrm{p}<0.001\right)$ than the pneumatic system.

For the pneumatic system, a pressure of $26 \mathrm{kPa}$, a delay of $155 \mathrm{~ms}$ and a layer height of $380 \mu \mathrm{m}$ were used. For the PCP, the same calibration data which were obtained in the preparation process were applied, i.e. calibration was not performed again but saved values used, a volume flow of $134.5 \mu \mathrm{l} / \mathrm{min}$ set, a delay of $284 \mathrm{~ms}$ and a layer height of $403 \mu \mathrm{m}$ used. The reduced layer height of $380 \mu \mathrm{m}$ for the pneumatic system was chosen to ensure that the strands of subsequent layers would be in direct contact with the previous layer when deposited. Due to the large standard deviation of the layer height for the pneumatic system $( \pm 75 \mu \mathrm{m})$ as compared to the one of the PCP $( \pm 35 \mu \mathrm{m})$ (Supplementary Figure $3 \mathrm{~K}, \mathrm{~L}$ ) using the same layer height for both systems, would risk that layers printed with the pneumatic system would not be in direct contact with the previously printed layer. Nonetheless, as the volume flow of the systems is independent of the layer 
A

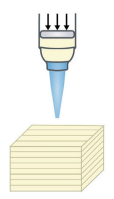

B

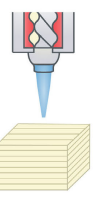

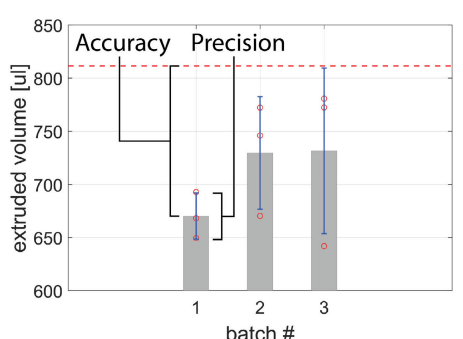

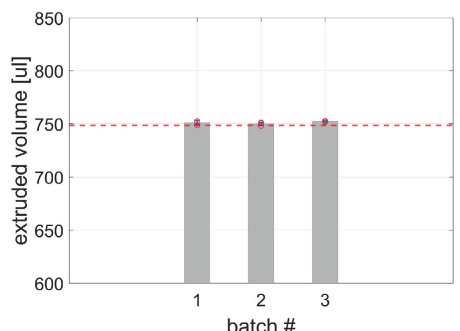

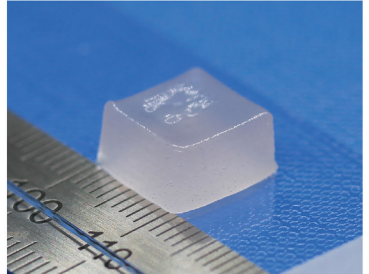

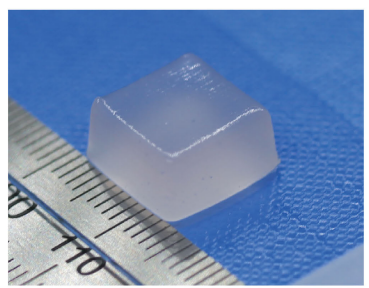

Figure $2 \mathrm{~A}$, Extruded volume of constructs printed with the pneumatic system. The picture shows a cube printed with the pneumatic system. A small defect can be seen on the surface on top of the cube. B, Extruded volume of constructs printed with the PCP. No defects were observed in the printed cubes. Red line: targeted volume. Mean $\pm S D, n=9$ (three batches), $\left({ }^{*} p<0.05,{ }^{* *} p<0.005, * * * p<0.001\right)$.

A

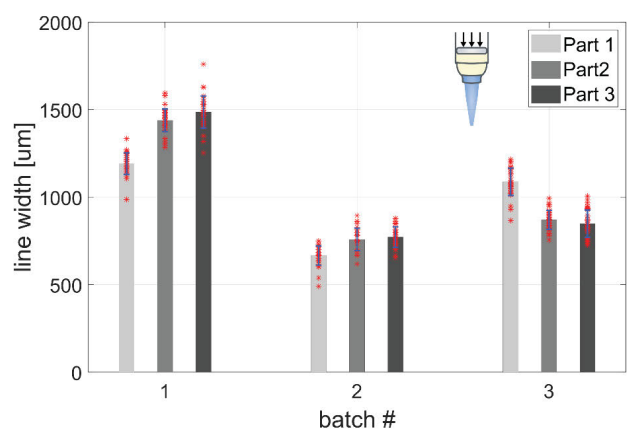

C

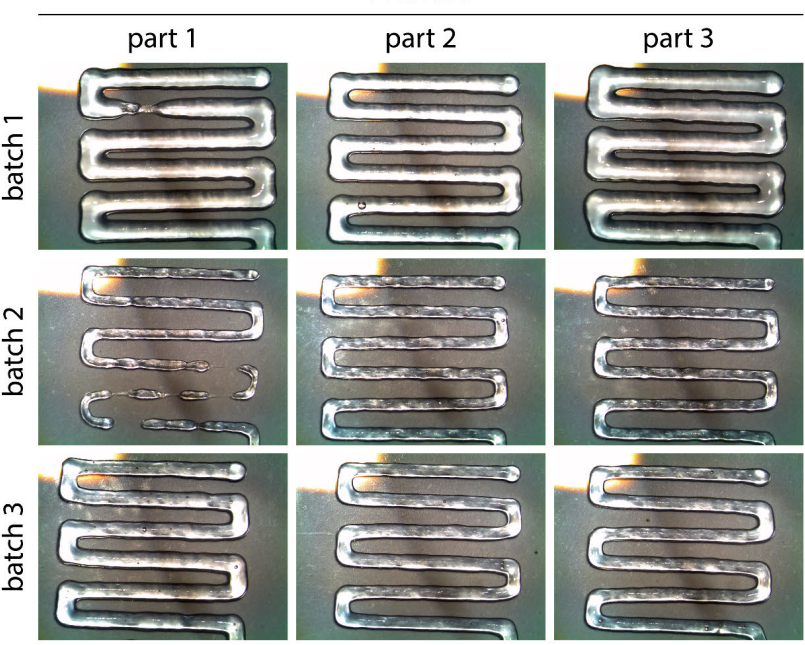

B
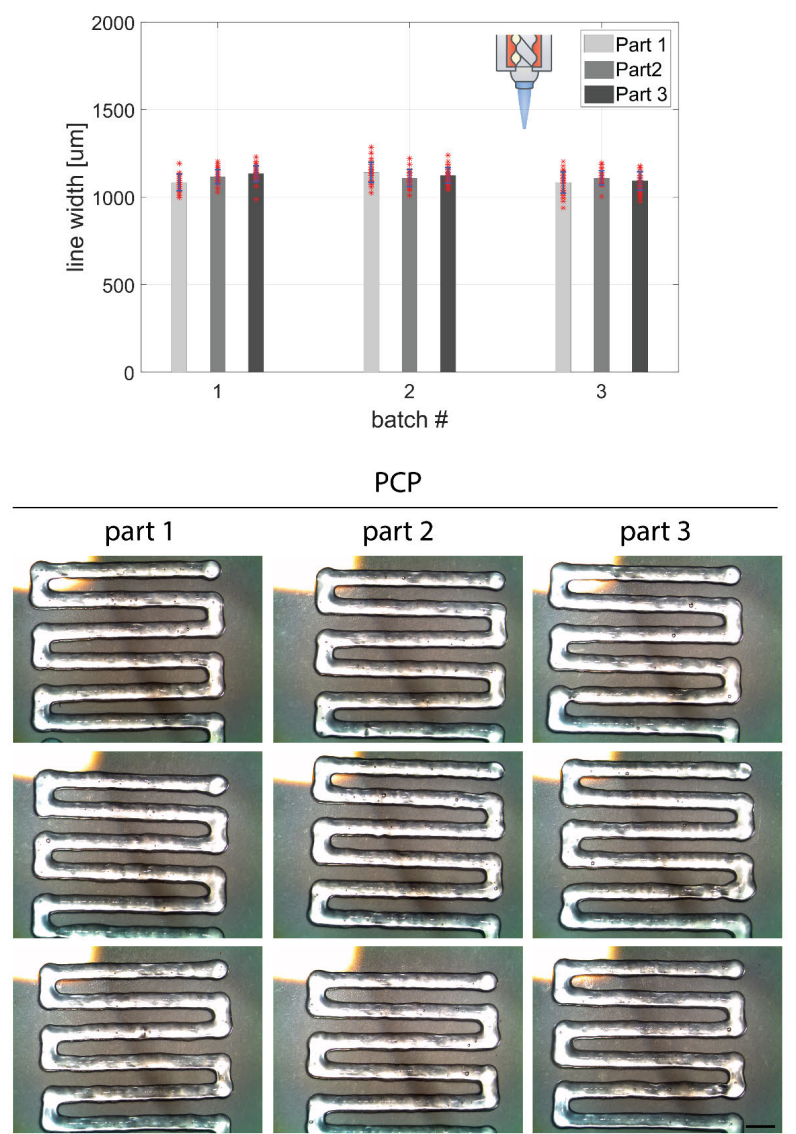

Figure 3 A-B, Line width of printed strands. Three batches of bioink were printed with either the pressure system (A) or the PCP (B) with different parts of the printing cartidge (part 1: initial part, part 2: middel part, part 3: end part). C, Pictures of the strands after extrusion. Scale bar: $2 \mathrm{~mm}$. 
A

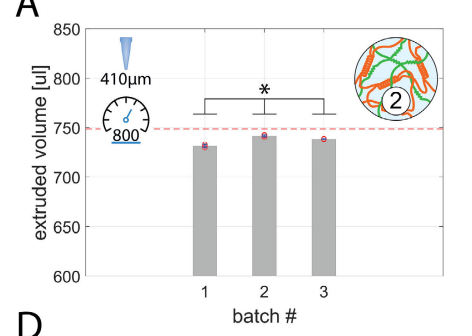

$\mathrm{D}$

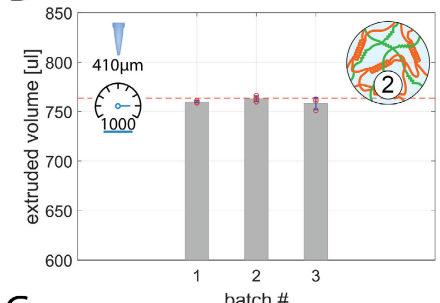

G

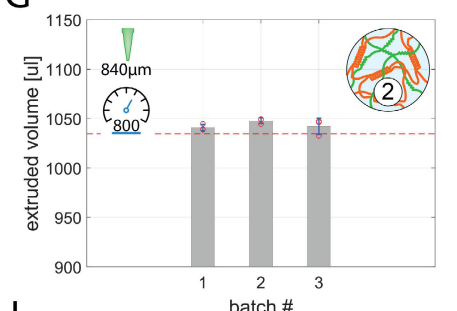

J

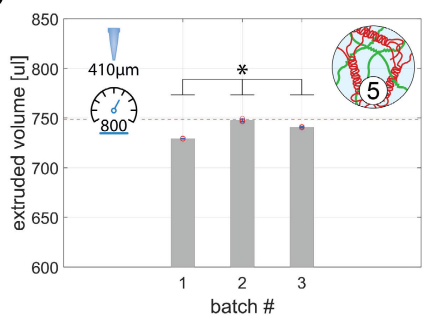

L

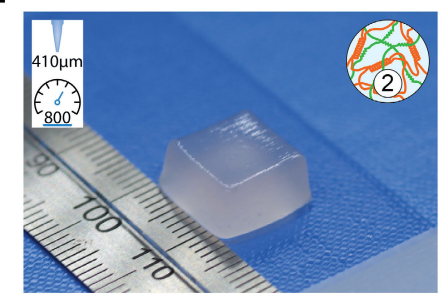

B

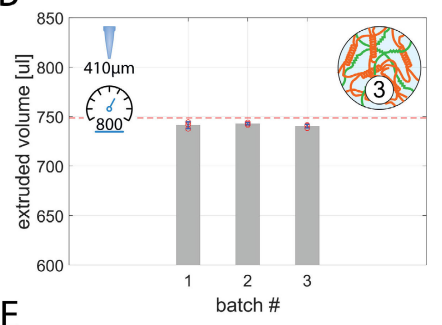

E

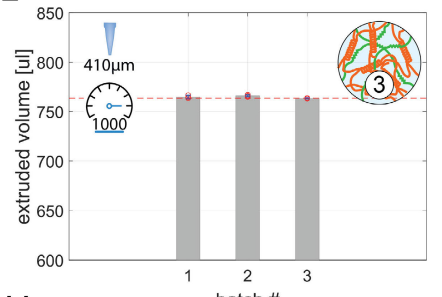

$\mathrm{H}$

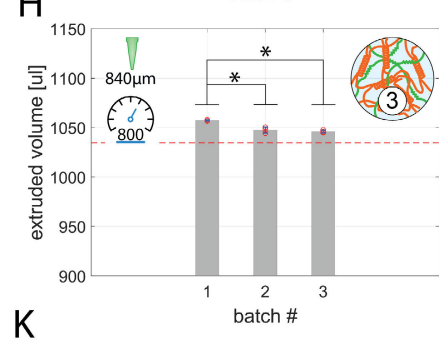

K

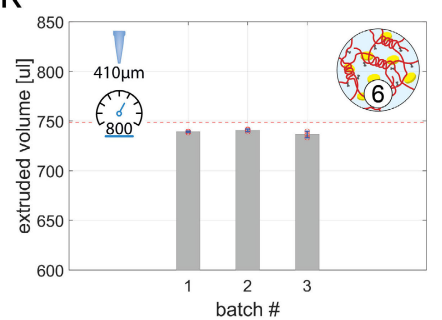

M

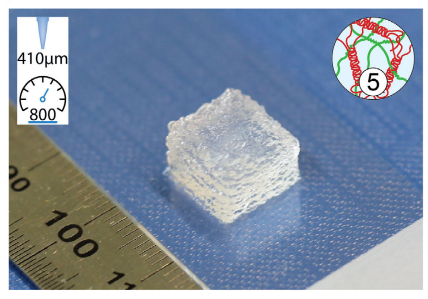

C

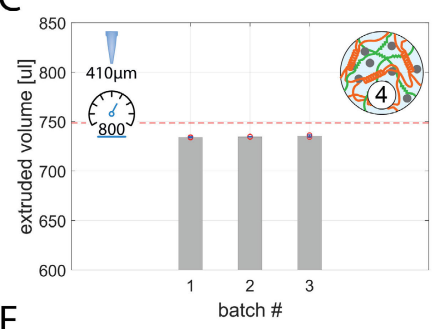

F

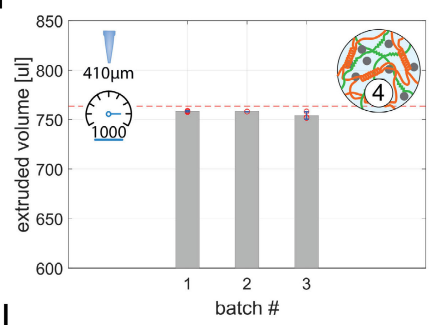

I

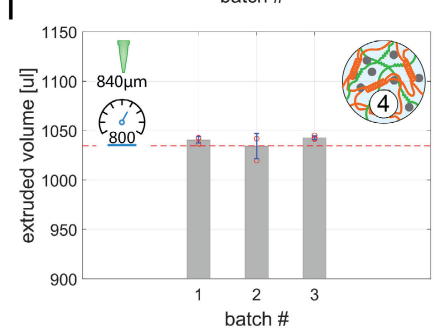

$\mathrm{N}$

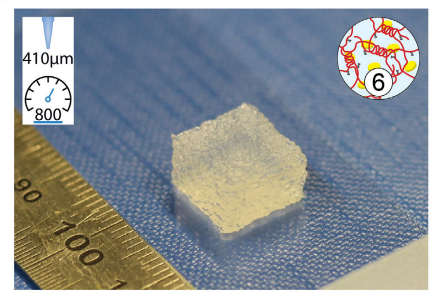

Figure 4 A-C, Extruded volume of cubes printed with a $410 \mu \mathrm{m}$ nozzle at $800 \mathrm{~mm} / \mathrm{min}$ for bioink 2-4 respectively. D-F, Extruded volume of cubes printed with a $410 \mu \mathrm{m}$ nozzle at $1000 \mathrm{~mm} / \mathrm{min}$ for bioink 2-4 respectively. G-I, Extruded volume of cubes printed with a $840 \mu \mathrm{m}$ nozzle at $800 \mathrm{~mm} / \mathrm{min}$ for bioink 2-4 respectively. Red line: targeted volume. J-K, Extruded volume of cubes printed with a $410 \mu \mathrm{m}$ nozzle at $400 \mathrm{~mm} / \mathrm{min}$ for the gelatin-alginate bioink and the GelMA-Laponite bioink respectively. L-N Representative images of cubes printed with the alginate-gellan gum bioink (Bioink 2), the gelatin bioink (Bioink 5) and the GelMA bioink (Bioink 6). All cubes were printed with the same settings with the PCP. Mean \pm SD, $n=9$ (three batches), $\left(* p<0.05, * * p<0.005,{ }^{* * *} p<0.001\right)$.

1 height, this difference might only influence the print 2 quality, e.g. by moving the nozzle tip through the top part of a printed layer.

The pneumatic system achieved an overall accuracy of $12.4 \pm 4.3 \%$ and a precision of $18.99 \pm 16.23 \mu \mathrm{l}$ (batch 1 : $17.39 \%$ and $12.56 \mu$ l, batch $2: 10.1 \%$ and $30.52 \mu$ l, batch 3: 9.8\% and $44.96 \mu \mathrm{l}$ ) but did clog twice, once for batch 2 8 and once for batch 3 (Figure $2 \mathrm{~A}$ ). When clogged, the
9 pressure had to be increased to extrude the material 10 clogging the nozzle or if the material would not extrude at 11 increased pressure, the nozzle would have to be replaced.

12 The PCP on the other hand achieved an overall accuracy 13 of $0.3 \pm 0.2 \%$ and a precision of $0.54 \pm 0.45 \mu \mathrm{l}$ (batch 1 : $140.3 \%$ and $1.12 \mu \mathrm{l}$, batch $2: 0.2 \%$ and $0.90 \mu \mathrm{l}$, batch 3 : $150.5 \%$ and $0.26 \mu \mathrm{l}$ ) without clogging (Figure $2 \mathrm{~B}$ ). Despite 16 the higher volume flow used for the pneumatic system, 
1 samples repeatedly showed a lower volume than the expected volume together with surface defects visible by small holes/missing material on the top surface of the cubes (Figure $2 \mathrm{~A}$ ).

Printing individual lines of bioink confirmed the differences in extrusion accuracy and precision between the two systems as the line width of lines printed with the pneumatic system showed a 5.6 times higher variation (PCP: $\pm 53 \mu \mathrm{m}$, pneumatic: $\pm 294 \mu \mathrm{m}$ ) and 22 times higher average batch to batch difference (PCP: $20 \mu \mathrm{m}$, pneumatic: $437 \mu \mathrm{m}$ ) compared to lines printed with the PCP (Figure 3).

\subsection{Influence of the bioink composition, printing speed and nozzle diameter on the accuracy and precision of the progressive cavity pump}

To further evaluate the accuracy and precision of the PCP, three different printing setups were used and evaluated with three different bioinks. Compared to the accuracy and precision of the pneumatic system, all setups used showed a significantly higher precision $\left({ }^{* *} \mathrm{p}<\right.$ $0.005)$ and accuracy $\left({ }^{* *} \mathrm{p}<0.005\right)$.

Cubes printed with a nozzle of $410 \mu \mathrm{m}$ and a feed rate of $800 \mathrm{~mm} / \mathrm{min}$, achieved an accuracy of $1.5 \pm 0.7 \%, 1.0$ $\pm 0.2 \%$ and $1.8 \pm 0.1 \%$ and a precision of $1.53 \pm 0.36 \mu \mathrm{l}$, $0.76 \pm 0.65 \mu \mathrm{l}$ and $0.28 \pm 0.27 \mu \mathrm{l}$ for Bioink 2,3 and 4 respectively (Figure $4 \mathrm{~A}-\mathrm{C}$ ). Increasing the feed rate to $1000 \mathrm{~mm} / \mathrm{min}$ but keeping the $410 \mu \mathrm{m}$ nozzle led to an accuracy of $0.4 \pm 0.3 \%, 0.2 \pm 0.2 \%$ and $0.9 \pm 0.3 \%$ and a precision of $1.35 \pm 1.48 \mu \mathrm{l}, 0.53 \pm 0.40 \mu \mathrm{l}$ and $0.91 \pm 1.09$ $\mu \mathrm{l}$ for Bioink 2, 3 and 4 respectively (Figure $4 \mathrm{D}-\mathrm{F}$ ). Lastly, changing the nozzle to an $840 \mu \mathrm{m}$ nozzle and keeping the federate at $800 \mathrm{~mm} / \mathrm{min}$ an accuracy of $0.9 \pm$ $0.4 \%, 1.5 \pm 0.6 \%$ and $0.4 \pm 0.4 \%$ and a precision of 1.86 $\pm 1.75 \mu \mathrm{l}, 1.82 \pm 0.68 \mu \mathrm{l}$ and $2.54 \pm 3.38 \mu \mathrm{l}$ were achieved for Bioink 2, 3 and 4 respectively (Figure $4 \mathrm{G}-\mathrm{I}$ ).

Accuracy and precision of the PCP did not show a significant dependency on the printing parameters, i.e. nozzle size and printing speed, nor on the material used and no significant difference was observed compared to the accuracy and precision of the cubes printed with Bioink 1.

\section{3.6 Printing of thermosetting bioinks}

Gelatin and its photocrosslinkable variant GelMA play a significant role in tissue engineering and bioprinting due to their similarity to the ECM of many tissues. (29) As gelatin and GelMA undergo thermal gelation during cooling, their extrusion generally requires either temperature-controlled extrusion, extrusion during a specific time window or the use of positive displacement extruder. $(25,26)$ To evaluate if the PCP can extrude such bioinks without temperature control, we printed three cubes per bioink batch. These cubes were printed consecutively and in contrast to the necessary adjustment of pressure to maintain adequate volume flow, no changes had to be performed with the PCP. Overall the PCP achieved an accuracy of $1.3 \pm 1.2 \%$ and a precision of $2.67 \pm 0.36 \mu \mathrm{l}$ for the gelatin bioink (Bioink 5) and $1.3 \pm$ $0.3 \%$ and $0.84 \pm 0.75 \mu$ l for the GelMA bioink (Bioink 6) (Figure $4 \mathrm{~J}, \mathrm{~K}$ and $\mathrm{M}, \mathrm{N}$ ). No time-dependency of the volume flow was observed throughout the experiment and no statistical difference to cubes printed with Bioink 2-4 and the different printing setups were found.

\subsection{Printing of complex structures}

The benefits of bioprinting are the creation of complex structures for which the bioprinter has to follow a complex printing path as defined in the g-code. Contrary to cubes which run on a single continuous print-path and only have a single start per layer, such complex shapes need the system to start and stop several times. To further characterize the accuracy and precision of the printing process we therefore printed ear and nose constructs (Figure $5 \mathrm{~A}, \mathrm{C}$ ). Despite these complex printing processes, the PCP could maintain its accuracy and precision with Bioink 2, 5 and 6 and achieve a 63 and 80 times higher precision than the pneumatic system.

The printing of these constructs was characterized by several start and stop signals for e.g. the perimeters and infill (Figure 5 B, D). We compared the pneumatic system against the PCP using Bioink 2. The PCP achieved a higher accuracy with a much smaller standard deviation (pneumatic: ear: $5.6 \pm 46.1 \%$, nose: $22.1 \pm 51.1 \%$ - PCP: ear: $2.5 \pm 0.7 \%$, nose: $3.1 \pm 0.6 \%$ ) and had a 63 and 80 times higher precision for the ear and nose respectively (pneumatic: ear: $396.49 \mu \mathrm{l}$, nose: $451.63 \mu \mathrm{l}$ - PCP: ear: $6.29 \mu \mathrm{l}$, nose: $5.65 \mu \mathrm{l})$. Only one print out of three which were printed with the pneumatic system achieved satisfactory results as indicated in the large uncertainty in precision (Figure $5 \mathrm{G}$ ). We further tested the gelatin-based bioinks as the printing time of these structures would generally require a pressure adjustment to maintain proper volume flow of these bioinks without thermal regulation of the printing process. (25) Despite printing cubes, ears and noses in a random order and therefore during different timepoints of the thermal gelation process, the PCP maintained its accuracy (gelatin: ear: $3.0 \pm 0.8 \%$, nose: $2.7 \pm 0.8 \%$ - GelMA: ear: $3.7 \pm 0.7 \%$, nose: $3.8 \pm 0.1 \%$ ) and precision (gelatin: ear: $6.95 \mu \mathrm{l}$, nose: $7.43 \mu \mathrm{l}-$ GelMA: ear: $5.68 \mu \mathrm{l}$, nose: $1.00 \mu \mathrm{l}$ ) (Figure $5 \mathrm{E}, \mathrm{F}$ ). 
A

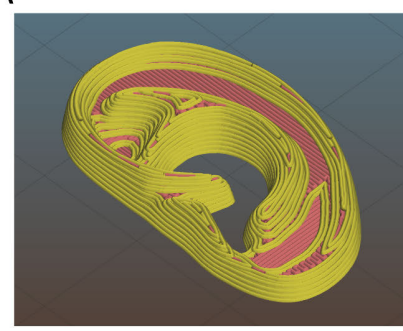

C

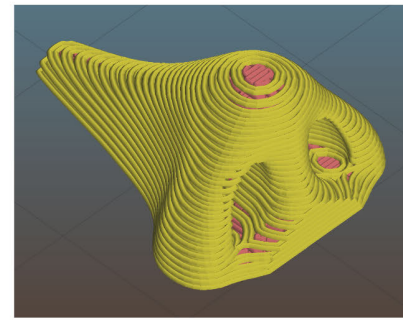

E

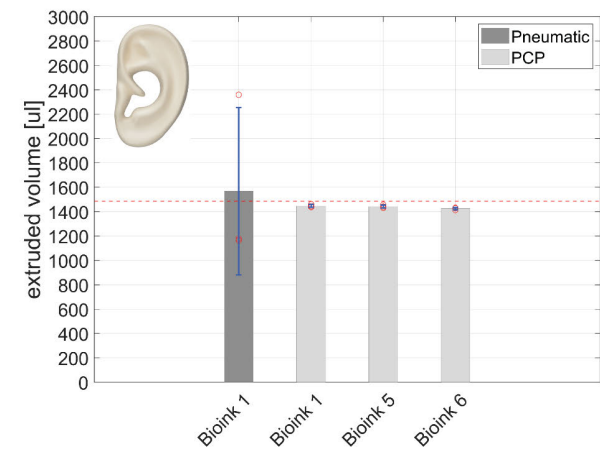

B

D
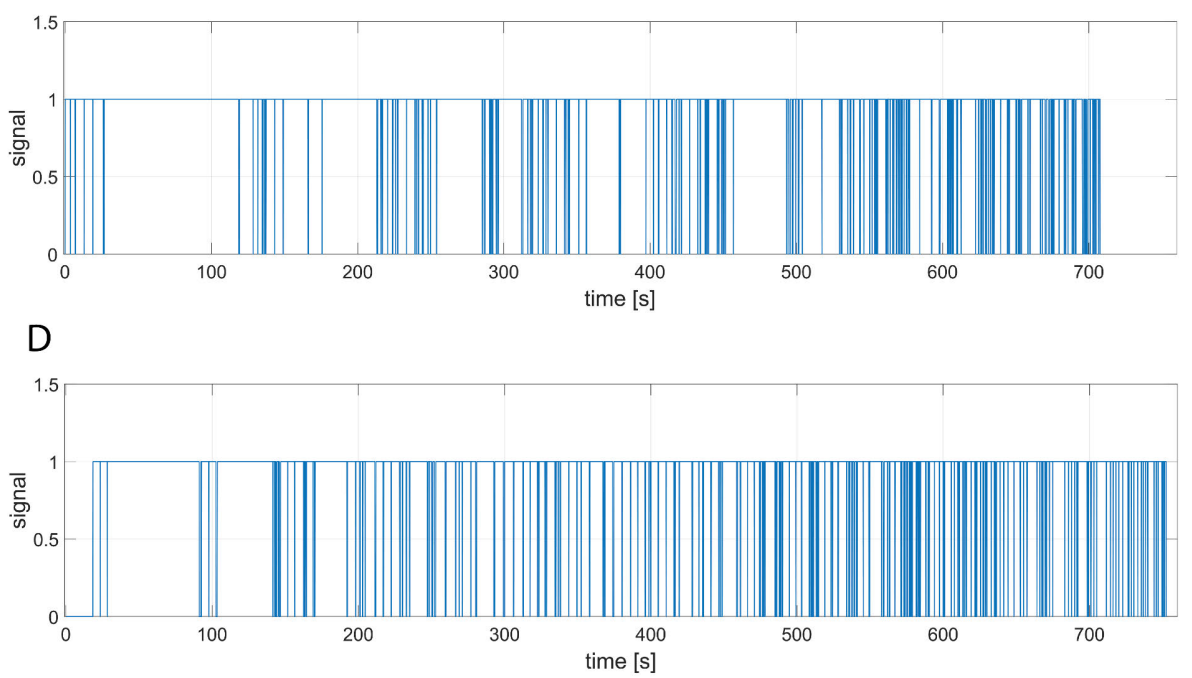

F

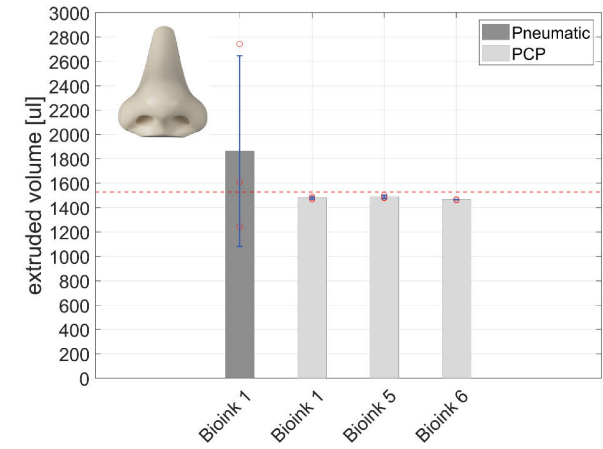

G
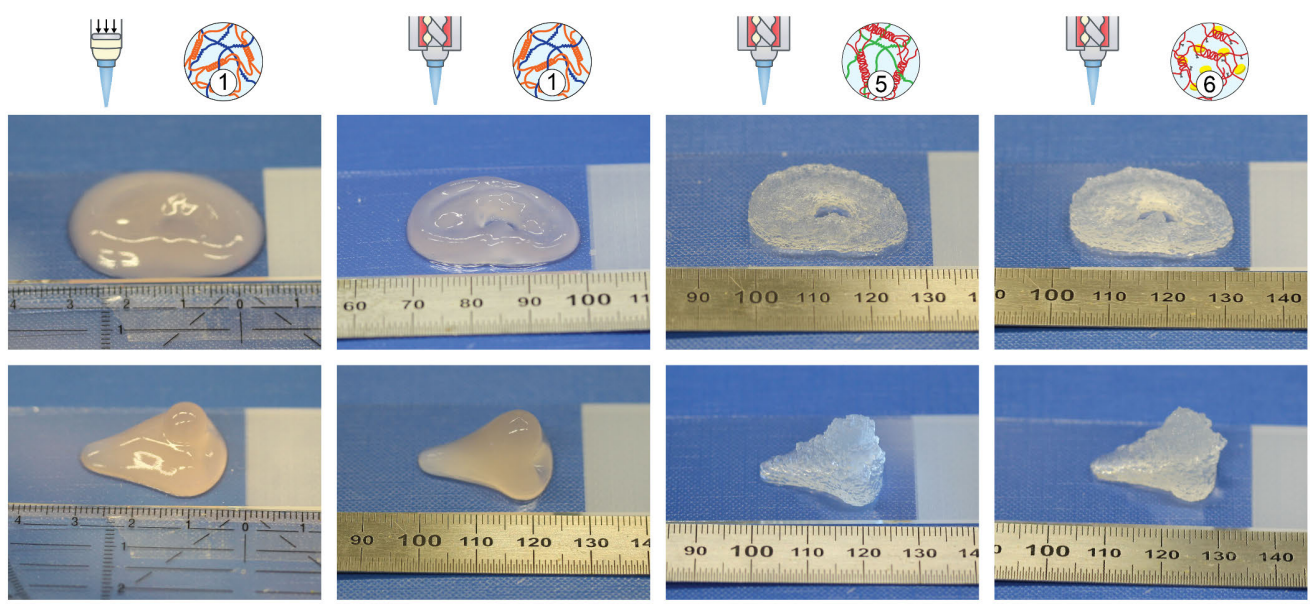

Figure 5 A, C, Printing path of the ear and nose model and B, D, signal recorded from the Biofactory bioprinter corresponding to the on (extrude: 1) and off (pause extrusion: 0 ) signal. E, F, Extruded volume of the ear and nose with the pneumatic system and the PCP printed with three different bioinks (Bioink 1, Bioink 5 and Bioink 6). G, images of the printed ears and noses with the different bioinks. Mean $\pm S D, n=3,(* p<0.05, * * p<0.005, * * * p<0.001)$. 
A
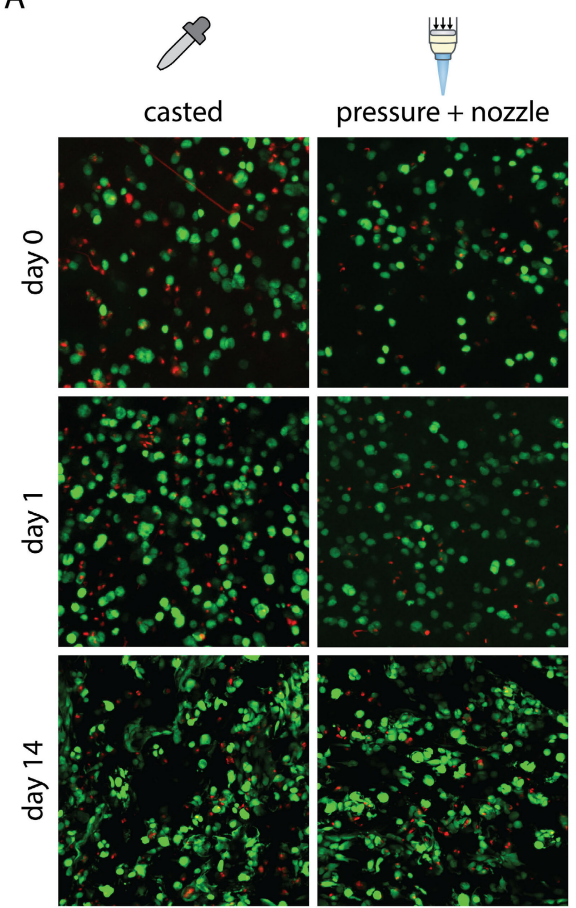

忨
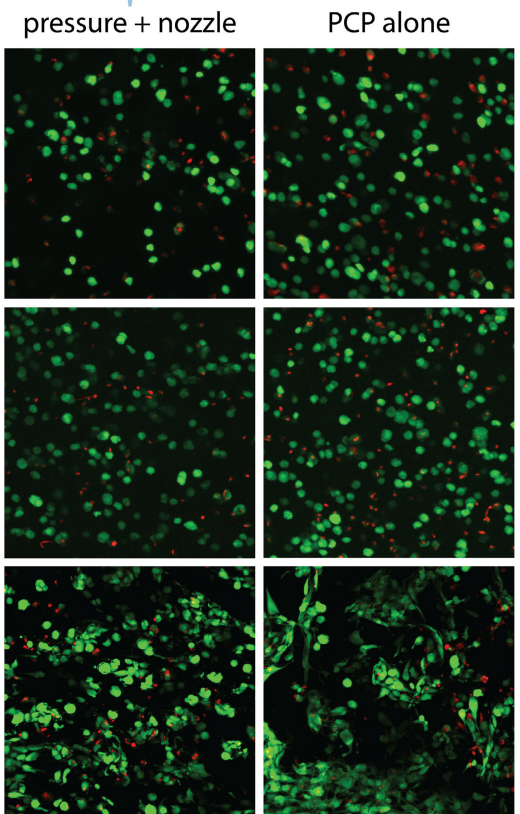

【L्य

PCP alone

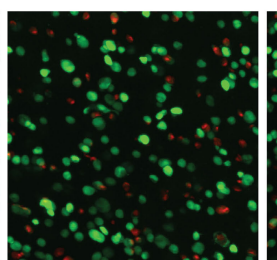

.
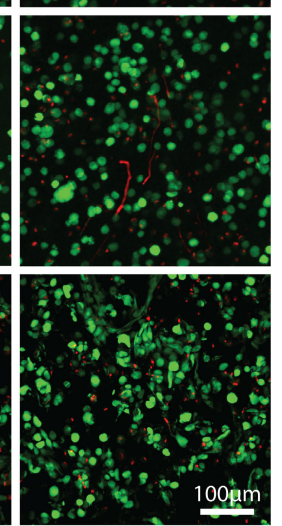

C

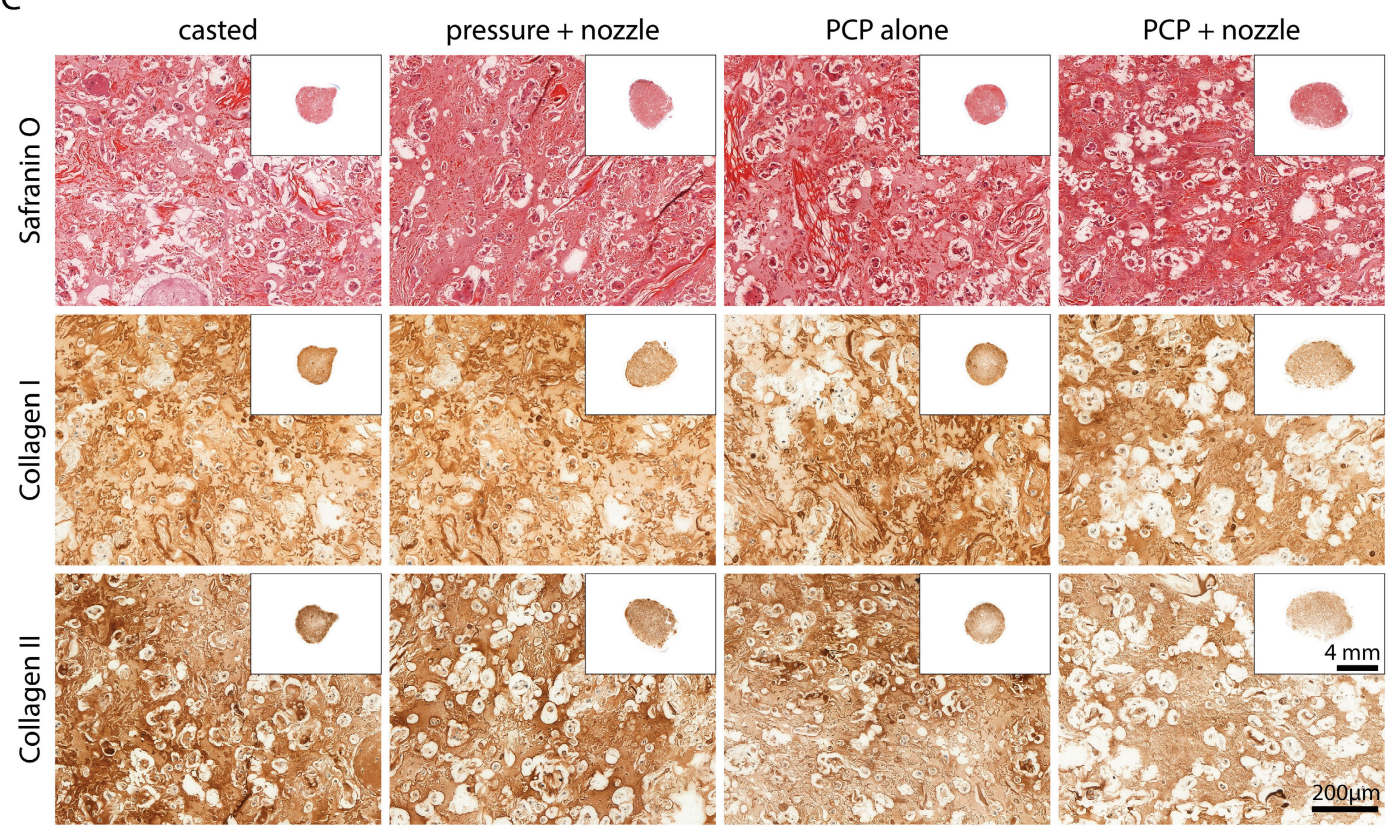

Figure 6 A, Z-projected images of the viability at day 0,1 and 14 . B, Viability of bovine chondrocytes at day 0,1 and 14 . Mean \pm SD, $n=3$, $\left({ }^{*} p<0.05,{ }^{* *} p<0.005,{ }^{* *} p<0.001\right)$. C, Histological and immunohistological analysis for GAGs (Safranin O), collagen I and collagen II.

\subsection{Viability and extracellular matrix deposition deposition}

The extrusion process in bioprinting can have a significant influence on cell viability due to shear forces experienced by cells. $(6,37)$ As the extrusion process through a PCP is more complex, we evaluated cell
7 viability for casted samples and compared it to the 8 viability of samples printed with the pneumatic system 9 with a $410 \mu \mathrm{m}$ nozzle, the PCP alone and the PCP with a $10410 \mu \mathrm{m}$ nozzle. Printing 3 million cells $/ \mathrm{ml}$ showed a drop 11 in cell viability when printed with the PCP and nozzle. 12 Nonetheless, at a cell density of 10 million cells/ml, no 13 difference between the extrusion methods was found and 
1 cell viability quickly recovered from the extrusion process.

In a first experiment, 3 million bovine chondrocytes were combined with the bioink. No evidence of an interaction effect of the method and timepoints (n.s.) and no dependence on the chosen timepoints (n.s.) was detected whereas the method significantly influenced viability $\left({ }^{* * *} \mathrm{p}<0.001\right)$. Samples extruded through the PCP alone (day 0: $77 \pm 2.7 \%$, day 1: $76 \pm 2.7 \%$, *p < 0.05) and through the PCP and a nozzle (day 0: $73 \pm 2.2$ $\%$, day 1: $75 \pm 2.3 \%$, ***p $<0.001$ ) showed a significantly lower viability compared to the casted samples (day 0: $80 \pm 3.1 \%$, day 1: $80 \pm 2.0 \%$ ). Samples bioprinted with the pneumatic system had a significantly higher viability (day 0: $80 \pm 3.2 \%$, p = 1, day 1: $79 \pm 1.9$ $\%, \mathrm{p}=1$ ) than samples extruded with the PCP and nozzle $\left({ }^{* *} \mathrm{p}<0.05\right)$ whereas no significant dependence was detected between samples extruded through the pneumatic system and the PCP alone (Supplementary Figure 5 A, B).

In a second experiment, the cell density was increased as previous studies showed that a higher cell density positively impacts chondrogenesis. (38) Additionally, cell viability was analyzed up to 14 days to evaluate the longterm viability and recovery of the cells from the extrusion process. First a decrease in viability was observed from day 0 to day $1(* * * p<0.001)$ which recovered after 14 days surpassing the initial viability $\left({ }^{* * *} \mathrm{p}<0.001\right)$. Overall, viability was significantly higher after 14 days compared to day 0 for the casted samples (day 0: $69.4 \pm$ 4.5\%, day 14: $82.9 \pm 0.7 \%$, $* * * p<0.001)$, samples extruded with the pneumatic system (day 0: $71.4 \pm 3.4 \%$, day 14: $\left.86.3 \pm 1.8 \%,{ }^{* * *} \mathrm{p}<0.001\right)$, with the PCP alone (day 0: $68.5 \pm 1.5 \%$, day 14: $78.4 \pm 4.8 \%$, *p $<0.05$ ) and PCP with nozzle (day 0: $70.0 \pm 3.8 \%$, day 14: $86.3 \pm$ $\left.2.4 \%,{ }^{* * *} \mathrm{p}<0.001\right)$. Contrary to the initial experiment with 3 million bovine chondrocytes, no significant differences were observed between the extrusion methods at the respective timepoints (Figure $6 \mathrm{~A}, \mathrm{~B}$ ).

Histological and immunohistological analysis of samples printed with 10 million bovine chondrocytes did not show any major differences between samples after 4 weeks (Figure $6 \mathrm{C}$ ). Cells deposited neocartilage components as indicated by the positive staining for GAGs (Safranin O) as well as collagen II and in addition to that, deposited collagen I.

\section{4. Discussion}

This study for the first time applied the PCP extrusion process to bioprinting of mammalian cells and compared the performance of a PCP to the pneumatic microextrusion system. The PCP outperformed the pneumatic system reaching a 41 times higher accuracy and 35 times higher precision. An explanation for this superior material deposition can be found considering that any inhomogeneities influence the material flow in the pneumatic system. Analyzing the flow rate of the pneumatic system (Supplementary Figure $3 \mathrm{C}$ ) it can be seen that a slight change in pressure already has a significant influence on the flow rate. Conversely, a slight change in the rheological properties of the bioink can have a significant influence on the flow rate when the pressure is kept constant. (39) These changes in rheological behavior could be observed within a single bioink batch and between batches (Supplementary Figure 2). Therefore, the flow rate is significantly altered during printing with the pneumatic system, leading to defects in the printed construct (Figure $4 \mathrm{~A}$ ). As the PCP functions based on the endless piston principle, creating an accurate and consistent flow by volumetric displacement of the created cavities, (13) such inhomogeneities do not influence the flow rate. Once the flow rate of the PCP is determined, it can reliably and reproducibly extrude the desired amount of material. Additionally, as the PCP continuously pushes new material into the nozzle and bioinks being largely incompressible, clogging events due to inhomogeneities will be pressed out if not larger than the nozzle diameter. In contrast, once the nozzle clogs when using the pneumatic system, only an increase in pressure can ensure continuous material deposition. (1, 11) Such clogging events were observed twice with the pneumatic system, leading to failed prints.

The ease of use of the PCP was further evaluated by excluding the entire printing preparation process and simply calibrating the material flow for the different bioinks. Slightly larger deviations in the accuracy were observed compared to constructs printed after performing the printing assessment. These deviations could potentially be related to imprecisions in performing the calibration of the PCP or to problems related to the layer integration. If during the calibration, the weight of the extruded material is underestimated, the PCP will later extrude too much material due to a higher flow rate. Regarding the layer integration, the bioink flow after extrusion influences the layer height of the construct which does not reach the exact layer height of 410/840 $\mu \mathrm{m}$. When the nozzle retracts, some of the material might not be integrated into the construct, and therefore pulled away together with the nozzle. Nonetheless, the accuracy and precision achieved with the PCP without performing the printing preparation process still outperformed the accuracy and precision achieved with the pneumatic system.

These results were further confirmed printing two thermosetting gelatin-based bioinks. As gelatin undergoes thermal gelation during cooling, its rheological properties 
change, ultimately requiring pressure adjustments when printed with a pneumatic system if a constant temperature cannot be maintained. $(25,26)$ We could show that despite these changes in rheological properties, the PCP can maintain its accuracy and precision, similar to the alginate-gellan gum bioink. These results could facilitate the use of thermosetting bioinks, removing the userdependent adjustment of the pressure when printed with the pneumatic system.

Bioprinting seldom relies on a continuous printing path as complex structures require the printing of perimeter and sophisticated infill structures. This leads to several occasions in which the bioprinting nozzle needs to be repositioned. During these processes, the material flow must stop and start again when the new position is reached. To demonstrate printing of such structures, ear and nose constructs were printed. Again, the PCP could outperformed the pneumatic extrusion process, showing a 63 and 80 times higher precision for the ear and nose respectively and could maintain this precision also for the thermosetting gelatin-based bioinks. The slightly larger standard error in printing ear and nose constructs compared to the cubes could arise from the longer printing time. Any imprecision in the calibration is aggravated the longer the print takes. Another factor contributing to this error could be the evaporation of water, which is larger, the longer the print takes. Nonetheless, these results underline the reliability of the PCP compared to the pneumatic system and show that despite changing rheological properties, the PCP maintains a constant volume flow.

No difference in viability between casted samples and samples printed with the pneumatic system for both conditions was observed. Previous studies showed a dependency of cell viability on the shear stresses experienced by cells during extrusion. $(37,40,41)$ These shear stresses are in turn dependent on the material and each cell type reacts differently to different levels of shear stresses. $(6,42)$ Two phenomena could have contributed to the preservation of cell viability between casted samples and samples printed with the pneumatic system. First, the viscosity of the shear thinning material might have dropped below a threshold under which bovine chondrocytes are not damaged. (42) This could further be influenced by the time bovine chondrocytes are exposed to these shear stresses. (37) Second, the viscoelastic properties of the bioink might protect cells from shear stresses during the extrusion process. (6) Nevertheless, as we observed a drop in viability before printing (casted samples), the mixing process of the cells with the bioink appears to influence viability more significantly than the extrusion process itself.
Depending on the cell concentration, a drop in viability of $3 \%$ was observed for the 3 million cells/ml condition but not for the 10 million cells/ml condition when extruded through the PCP alone. As PCPs use a pushingand-suction action and therefore exert low shear rates on the material, shear stresses experienced by the cells alone might not explain the drop in viability. In addition to these shear stresses, the seal-lines where stator and rotor meet to form a tight junction should be considered. At these junctions cells trapped between stator and rotor will be damaged, thereby contributing to the drop in viability. Lastly, the drop in viability of $7 \%$ after extrusion through the PCP with a $410 \mu \mathrm{m}$ nozzle for the 3 million cells $/ \mathrm{ml}$ condition could be a combined effect of the damage experienced by the cells when travelling through the PCP and then being pushed through the nozzle. Cells, whose cell membrane is damaged by extrusion through the PCP could be more susceptible to the shear stresses experienced when extruded through the nozzle. $(37,42)$ Similar to the extrusion through the PCP without nozzle, this effect might diminish with higher cell densities. To confirm these assumptions, simulations of the flow within the PCP would be necessary together with exposing the cells to controlled shear conditions.

Neocartilage formation was observed in all conditions to a similar extend. Positive stainings for GAGs and collagen II underline the chondrogenic potential of chondrocytes embedded in the bioink. Contrary to the ECM of native cartilage (Supplementary Figure 6) collagen I deposition could be related to the dedifferentiation of chondrocytes in 2D culture as often seen in tissue engineered cartilage. (43)

Overall, our results are in accordance with the study of Ning et al. who could show that upon increasing cell density, an increase in cell viability could be observed. (42) Further, despite exposing cells to this complex extrusion environment, no major drop in viability was observed and the overall cell viability was well within previously reported values of $50-90 \%$ (26, 39, 44-46). Importantly, cells recover from the preparation/extrusion process and reached viabilities of around $85 \%$ after 14 days. As demonstrated by Kesti et al. such a recovery in cell viability ultimately leads to the formation of cartilaginous ECM. (23) These results indicate that despite the more complex extrusion process, PCPs are a valuable tool for bioprinting, supporting the printing of mammalian cells. Of note is that the PCP employed in this study was developed for industrial applications such as the dispensing of silicones and adhesives. Achieving similar cell viabilities as the pneumatic system or casting while providing direct control over the extrusion process represents a significant improvement in the bioprinting process. 
Despite the advantages of direct control over volume flow, pneumatic extrusion still remains the most commonly used extrusion process. Nonetheless, other processes such as piston-driven extrusion and auger screws are gaining interest. $(6,26,47)$ These positive displacement extrusion mechanisms overcome one of the biggest hurdles of pneumatic extrusion. Without knowledge of the exact rheological properties of the bioink, the volume flow cannot be predicted for the pneumatic system. (39, 40, 48) Each batch of bioink would require rheological characterization before usage, making this procedure extremely time-consuming. With the addition of PCPs to the toolset of positive displacement mechanisms, three approaches to directly control volume flow now exist: i) piston-driven extrusion, ii) screw-driven extrusion and iii) PCPs.

Piston-driven extrusion is possibly closest to pneumatic extrusion as no additional complexity is added to the extrusion process. Similar to pneumatic extrusion, the material is extruded by a force acting on a plunger and the pressure developed for the same volume flow is equivalent. Cells therefore experience similar shear stresses during extrusion. A drawback is that each cartridge would require a special adapter to be mounted in such an extruder. In screw-driven extrusion, cells experience additional shear stresses during their flow through the screw. Ning et al. could show that for bioinks without yield point, such as alginate, these stresses cause a drop in viability of about $4.5 \%$. (6) In the present study we could show that PCPs are able to maintain an accurate and precise volume flow and that their effect on cell viability is minor. In contrast to the study of Ning et al., our study focused more extensively on the reliability and reproducibility of PCPs using bioinks with a yield point. A drawback of screw-driven and PCP extrusion is that cells and material come in contact with parts of the extruder which need to be sterilized. PCPs compared to screw-driven extrusion displace the material via a pushing-and-suction action. Instead of shearing the material in the created cavities, the seal-lines function as infinite plunger pushing the material towards the discharge end of the pump. Thereby PCPs possibly exert lower shear stresses compared to screw-driven extruders. A major concern of PCPs are the seal-lines between rotor and stator in which cells might be trapped and damaged. Similar to the optimization of PCPs for the oil industry, measures could be taken to optimize the geometry to reduce cell damage. (49) To reliably compare the three systems, a study with different bioinks, cell types and all three extrusion methods would be required.

Limitations of this work are the comparison of the pneumatic extrusion process to PCPs without direct comparison to other volumetric extrusion processes. We aimed to introduce PCPs to the field of bioprinting, but it should be noted that with more sophisticated PCPs developed specifically for bioprinting, such comparisons will become essential. Further, only one cell type was tested as a model cell type and with bioprinting PCPs a more in-depth characterization of the effect of PCPs on cell viability and behaviour will be necessary.

Future work will rely on the redesign of PCPs specifically for bioprinting to improve cell viability while maintaining their reliability and reproducibility. A major drawback of the system used in this study was the large dead volume needed to fill the cavity before the rotor/stator. As materials used for bioprinting can be costly, a reduction in dead volume of these pumps will be essential. Additionally, to be able to print finer structures with smaller nozzles, a reduction in the minimum volume flow will be essential as the PCP used in this study supports a minimum volume flow of $120 \mu \mathrm{l} / \mathrm{min}$. Using a feedrate of $800 \mathrm{~mm} / \mathrm{min}$, a nozzle of $200 \mu \mathrm{m}$ diameter would require a volume flow of $32 \mu \mathrm{l} / \mathrm{min}$. To reduce the increase in shear stresses experienced by cells due to the smaller nozzle size, printing at smaller feedrates can be performed which in turn would require an even lower volume flow. From a translational perspective, all materials in contact with cellular materials should either be easily cleanable or single-use. With advances in the extrusion methods and the optimization of screw-driven and PCP extrusion for bioprinting, a comparison between the three different volumetric processes should be carried out to obtain a direct comparison of their merits and drawbacks, which would further help researchers to choose the best system for their bioprinting process.

\section{Conclusion}

In summary, progressive cavity pumps offer a valuable tool to significantly improve precision and accuracy of micro-extrusion bioprinting. We could show that a 41 times higher accuracy and up to 80 times higher precision could be achieved when using PCPs compared to pneumatic extrusion. Good cell viability was observed when extruding cell-laden bioinks through PCPs. The capability of PCPs to extrude and maintain a given flow rate irrespective of the bioink's rheological properties will simplify implementation of more complex, multifunctional bioprinting materials. Particularly clinical translational of bioprinting, which depends heavily upon standardization and validation, would highly benefit from such a precise and accurate extrusion process.

\section{Acknowledgements}

This work was supported by the Swiss National Science Foundation (CRSII5_173868 to MZW). The authors 
1 would like to thank Annemarie Brandstetter and Raphael Lichtnecker from ViscoTec GmbH for the fruitful discussions, Dr. Nicolas Broguière for help with the rheology and Dr. Emma Cavalli for help with the cell culture.

\section{References}

1. Vijayavenkataraman S, Yan WC, Lu WF, Wang $\mathrm{CH}$, Fuh JYH. 3D bioprinting of tissues and organs for regenerative medicine. Adv Drug Deliv Rev. 2018;132:296-332.

2. Wilson WC, Boland T. Cell and organ printing 1: Protein and cell printers. Anat Rec Part A. 2003;272a(2):491-6.

3. Murphy SV, Atala A. 3D bioprinting of tissues and organs. Nat Biotechnol. 2014;32(8):773-85.

4. Pereira FDAS, Parfenov V, Khesuani YD, Ovsianikov A, Mironov V. Commercial 3D Bioprinters. In: Ovsianikov A, Yoo J, Mironov V, editors. 3D Printing and Biofabrication. Cham: Springer International Publishing; 2018. p. 535-49.

5. Gillispie G, Prim P, Copus J, Fisher J, Mikos AG, Yoo JJ, et al. Assessment methodologies for extrusion-based bioink printability. Biofabrication. 2020;12(2):022003.

6. Ning L, Yang B, Mohabatpour F, Betancourt N, Sarker MD, Papagerakis P, et al. Process-induced cell damage: pneumatic versus screw-driven bioprinting. Biofabrication. 2020;12(2):025011.

7. Groll J, Burdick JA, Cho DW, Derby B, Gelinsky M, Heilshorn SC, et al. A definition of bioinks and their distinction from biomaterial inks. Biofabrication. 2018;11(1):013001.

8. Valkenaers H, Vogeler F, Voet A, Kruth J-P, editors. Screw extrusion based $3 \mathrm{D}$ printing, a novel additive manufacturing technology. Proceedings of the 5th international conference on competitive manufacturing; 2013: University of Stellenbosch.

9. Visser J, Peters B, Burger TJ, Boomstra J, Dhert WJ, Melchels FP, et al. Biofabrication of multi-material anatomically shaped tissue constructs. Biofabrication. 2013;5(3):035007.

10. Chimene D, Lennox KK, Kaunas RR, Gaharwar AK. Advanced Bioinks for 3D Printing: A Materials Science Perspective. Ann Biomed Eng. 2016;44(6):2090102.

11. Müller M, Fisch P, Molnar M, Eggert S, Binelli M, Maniura-Weber K, et al. Development and thorough characterization of the processing steps of an ink for 3D printing for bone tissue engineering. Materials Science and Engineering: C. 2020;108:110510.

12. Murphy SV, De Coppi P, Atala A. Opportunities and challenges of translational 3D bioprinting. Nat Biomed Eng. 2020;4(4):370-80.

13. Nelik L. Centrifugal \& rotary pumps: fundamentals with applications: CRC Press; 1999.
14. La C. Progressive Cavity Pump Systems: When Precision and Repeatability are Vital. Global SMT \& Packaging. 2017:23-5.

15. Hart F. How Microdispensing Technology Is Changing Medical Device Manufacturing. Medical Design Briefs. 2017:24-6.

16. Kokkinis D, Schaffner M, Studart AR. Multimaterial magnetically assisted 3D printing of composite materials. Nat Commun. 2015;6:8643.

17. Kokkinis D, Bouville F, Studart AR. 3D Printing of Materials with Tunable Failure via Bioinspired Mechanical Gradients. Adv Mater. 2018;30(19):e1705808.

18. Saveth KJ, Klein ST. The Progressing Cavity Pump: Principle and Capabilities. SPE Production Operations Symposium; 1989/1/1/; Oklahoma City, Oklahoma. SPE: Society of Petroleum Engineers; 1989. p. 429-34.

19. Vetter G, Wirth W, editors. Understand Progressing Cavity Pumps Characteristics And A Void Abrasive Wear. Proceedings of the 12th international pump users symposium; 1995: Turbomachinery Laboratories, Department of Mechanical Engineering, Texas A\&M.

20. Yao LN, Ou JF, Wang GY, Cheng CY, Wang W, Steiner H, et al. bioPrint: A Liquid Deposition Printing System for Natural Actuators. 3d Print Addit Manuf. 2015;2(4):169-79.

21. Wang G, Yao L, Wang W, Ou J, Cheng C-Y, Ishii H, editors. xPrint: A Modularized Liquid Printer for Smart Materials Deposition. Proceedings of the 2016 CHI Conference on Human Factors in Computing Systems; 2016; San Jose, California, USA: Association for Computing Machinery.

22. Schaffner M, Ruhs PA, Coulter F, Kilcher S, Studart AR. 3D printing of bacteria into functional complex materials. Sci Adv. 2017;3(12):eaao6804.

23. Kesti M, Eberhardt C, Pagliccia G, Kenkel D, Grande D, Boss A, et al. Bioprinting Complex Cartilaginous Structures with Clinically Compliant Biomaterials. Adv Funct Mater. 2015;25(48):7406-17.

24. Matti K, Philipp F, Marco P, Edoardo M, Marcy Z-W. Guidelines for standardization of bioprinting: a systematic study of process parameters and their effect on bioprinted structures. BioNanoMaterials. 2016;17(34):193-204.

25. Jiang T, Munguia-Lopez JG, Gu K, Bavoux MM, Flores-Torres S, Kort-Mascort J, et al. Engineering bioprintable alginate/gelatin composite hydrogels with tunable mechanical and cell adhesive properties to modulate tumor spheroid growth kinetics. Biofabrication. 2019;12(1):015024.

26. Cidonio G, Alcala-Orozco CR, Lim KS, Glinka M, Mutreja I, Kim Y-H, et al. Osteogenic and angiogenic tissue formation in high fidelity nanocomposite Laponitegelatin bioinks. Biofabrication. 2019;11(3):035027. 
27. Morris ER, Nishinari K, Rinaudo M. Gelation of gellan - A review. Food Hydrocolloid. 2012;28(2):373411.

28. Mouser VH, Melchels FP, Visser J, Dhert WJ, Gawlitta D, Malda J. Yield stress determines bioprintability of hydrogels based on gelatinmethacryloyl and gellan gum for cartilage bioprinting. Biofabrication. 2016;8(3):035003.

29. Yue K, Trujillo-de Santiago G, Alvarez MM, Tamayol A, Annabi N, Khademhosseini A. Synthesis, properties, and biomedical applications of gelatin methacryloyl (GelMA) hydrogels. Biomaterials. 2015;73:254-71.

30. Nichol JW, Koshy ST, Bae H, Hwang CM, Yamanlar S, Khademhosseini A. Cell-laden microengineered gelatin methacrylate hydrogels. Biomaterials. 2010;31(21):5536-44.

31. Ojansivu M, Rashad A, Ahlinder A, Massera J, Mishra A, Syverud K, et al. Wood-based nanocellulose and bioactive glass modified gelatin-alginate bioinks for 3D bioprinting of bone cells. Biofabrication. 2019;11(3):035010.

32. Paxton N, Smolan W, Bock T, Melchels F, Groll J, Jungst T. Proposal to assess printability of bioinks for extrusion-based bioprinting and evaluation of rheological properties governing bioprintability. Biofabrication. 2017;9(4):044107.

33. Li X, Chen S, Li J, Wang X, Zhang J, Kawazoe N, et al. 3D Culture of Chondrocytes in Gelatin Hydrogels with Different Stiffness. Polymers (Basel). 2016;8(8):269.

34. Comminal R, Serdeczny MP, Pedersen DB, Spangenberg J. Numerical Modeling of the Material Deposition and Contouring Precision in Fused Deposition Modeling. 29th Annual International Solid Freeform Fabrication Symposium (SFF Symp 2018): Laboratory for Freeform Fabrication; 2018. p. 1855-64.

35. Mezger TG. The Rheology Handbook. 4th ed: Vincentz Network; 2014.

36. Koopmans RJ. Die swell or extrudate swell. In: Karger-Kocsis J, editor. Polypropylene: An A-Z reference. Dordrecht: Springer Netherlands; 1999. p. 15862.

37. Blaeser A, Duarte Campos DF, Puster U, Richtering W, Stevens MM, Fischer H. Controlling Shear Stress in 3D Bioprinting is a Key Factor to Balance Printing Resolution and Stem Cell Integrity. Adv Healthc Mater. 2016;5(3):326-33.

38. Mauck RL, Wang CC, Oswald ES, Ateshian GA, Hung CT. The role of cell seeding density and nutrient supply for articular cartilage tissue engineering with deformational loading. Osteoarthritis Cartilage. 2003;11(12):879-90.

39. Emmermacher J, Spura D, Cziommer J, Kilian D, Wollborn T, Fritsching U, et al. Engineering considerations on extrusion-based bioprinting: interactions of material behavior, mechanical forces and
58 cells in the printing needle. Biofabrication. 59 2020;12(2):025022.

$6040 . \quad$ Li M, Tian X, Schreyer DJ, Chen X. Effect of 61 needle geometry on flow rate and cell damage in the 62 dispensing-based biofabrication process. Biotechnol 63 Prog. 2011;27(6):1777-84.

64 41. Muller M, Ozturk E, Arlov O, Gatenholm P, 65 Zenobi-Wong M. Alginate Sulfate-Nanocellulose Bioinks for Cartilage Bioprinting Applications. Ann Biomed Eng. 2017;45(1):210-23.

42. Ning L, Guillemot A, Zhao J, Kipouros G, Chen $\mathrm{X}$. Influence of Flow Behavior of Alginate-Cell Suspensions on Cell Viability and Proliferation. Tissue Engineering Part C: Methods. 2016;22(7):652-62.

43. Chung C, Mesa J, Miller GJ, Randolph MA, Gill TJ, Burdick JA. Effects of Auricular Chondrocyte Expansion on Neocartilage Formation in Photocrosslinked Hyaluronic Acid Networks. Tissue Engineering. 2006;12(9):2665-73.

44. Mendes BB, Gomez-Florit M, Hamilton AG, Detamore MS, Domingues RMA, Reis RL, et al. Human platelet lysate-based nanocomposite bioink for bioprinting hierarchical fibrillar structures. Biofabrication. 2019;12(1):015012.

45. Jessop ZM, Al-Sabah A, Gao N, Kyle S, Thomas B, Badiei N, et al. Printability of pulp derived crystal, fibril and blend nanocellulose-alginate bioinks for extrusion 3D bioprinting. Biofabrication. 2019;11(4):045006.

46. Idaszek J, Costantini M, Karlsen TA, Jaroszewicz J, Colosi C, Testa S, et al. 3D bioprinting of hydrogel constructs with cell and material gradients for the regeneration of full-thickness chondral defect using a microfluidic printing head. Biofabrication. 2019;11(4):044101.

47. Ning L, Chen X. A brief review of extrusionbased tissue scaffold bio-printing. Biotechnol J. 2017;12(8).

48. Suntornnond R, Tan EYS, An J, Chua CK. A Mathematical Model on the Resolution of Extrusion Bioprinting for the Development of New Bioinks. Materials. 2016;9(9):756.

49. Zhou XZ, Shi GC, Cao G, Sun CL, He Y, Liu H, et al. Three dimensional dynamics simulation of progressive cavity pump with stator of even thickness. Journal of Petroleum Science and Engineering. 2013;106:71-6. 